\title{
High-Fidelity Aeroelastic Computations of a Flapping Wing with Spanwise Flexibility
}

\author{
Raymond E. Gordnier * \\ Air Force Research Laboratory, Wright-Patterson AFB, OH 45433-7512

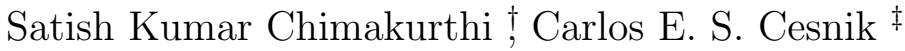 \\ University of Michigan, Ann Arbor, MI \\ Peter J. Attar $\S$ \\ University of Oklahoma, Norman, OK
}

\begin{abstract}
A high-order (up to 6th order) Navier-Stokes solver is coupled with a structural solver that decomposes the equations of three-dimensional elasticity into cross-sectional, smalldeformation and spanwise, large-deformation analyses for slender wings. The resulting high-fidelity aeroelastic solver is applied to the investigation of rigid, moderately flexible and highly flexible rectangular wings undergoing a pure plunging motion. Comparisons of the computed results with available experimental measurements demonstrate good agreement. A description of the complex interaction between the unsteady aerodynamics and the flexible wing structural dynamics is given. Connections between the results of this analysis and enhanced loads for the moderately flexible wing are made. Results presented suggest that an optimum amount of flexibility exists for the case of a plunging wing and is associated with wing motions where the wing tip deflection and wing root motion are in phase over much of the plunge cycle.
\end{abstract}

\section{Introduction}

The flexible flapping wing features a number of attractive characteristics that have driven interest in their application to micro air vehicle (MAV) development. The flapping wing is able to simultaneously provide both lift and thrust for the vehicle. The inherent flexibility of the structure may be used to adapt to unsteady aerodynamic loadings (gusts and crosswinds) and provide for very agile flight maneuvers. Flapping wing MAVs also have the potential to effectively meet the requirements for both hovering and perching.

The highly nonlinear aerodynamics and unsteady aero/structural coupling present a host of technical challenges to be overcome when simulating a flapping wing MAV design. MAVs operate at low Reynolds numbers requiring a unified computational approach for laminar, transitional, and turbulent flows. Coupling of transition with the surface motion and deformation further complicates the problem. Highly unsteady vortex generation and vortex interactions dominate the nonlinear aerodynamic behavior and are strongly linked to the flapping motion and flexibility. Unconventional structural materials and layout as well as large structural deflections give rise to highly nonlinear structural behavior limiting the applicability of conventional linear modal structural modeling.

While a significant body of work has been undertaken to understand the aerodynamics and structural dynamics of MAVs (see References 1-3) there is still much to be understood about the aerodynamics of flapping flight and the aeroelasticity of flapping wings. Recently, Heathcote et al. ${ }^{4}$ conducted experimental measurements of a rectangular wing with NACA0012 airfoil section oscillating in pure heave as a canonical problem to investigate the effect of spanwise flexibility on thrust, lift and propulsive efficiency of flapping

\footnotetext{
* Senior Research Aerospace Engineer, Computational Sciences Branch, Air Vehicles Directorate, Associate Fellow, AIAA

${ }^{\dagger}$ Post Doctoral Research Fellow, Department of Aerospace Engineering, University of Michigan, Ann Arbor, MI 48109, Currently Multiphysics Software Developer, ANSYS Inc, Canonsburg, PA

$\ddagger$ Professor, Department of Aerospace Engineering, University of Michigan, Ann Arbor, MI 48109, Associate Fellow, AIAA

$\S$ Assistant Professor, Department of Mechanical and Aerospace Engineering, Member, AIAA
} 
wings. Tang et al., ${ }^{5}$ Chimakurthi et al. ${ }^{6}$ Aono et al. ${ }^{7}$ and Chimakurthi ${ }^{8}$ have developed a computational aeroelasticity framework to analyze flexible flapping wings for MAVs. Their computational framework couples a low order (2nd order) Navier-Stokes solver with a structural solver UM/NLABS which decomposes the equations of 3-D elasticity into a linear cross-sectional and nonlinear spanwise analyses for slender wings. They then applied this solver to the plunging wing problem investigated by Heathcote et al.

In the present paper a high-order (up to 6th order) implicit large eddy simulation (ILES) solver for the Navier-Stokes equations is coupled with the UM/NLABS structural solver. This aerodynamic solver has been shown to successfully simulate the mixed laminar/transitional/turbulent vortical flows that dominate the aerodynamics of these low Reynolds number problems (see for instance References 9 and 10). Computations are performed with the new high-order aeroelastic solver for the flexible and highly flexible flapping wing cases investigated experimentally by Heathcote et al. ${ }^{4}$ and computationally in References $5-7$. A description of the resulting transitional flow over the dynamic, flexible wing as well as its its impact on the coupled fluidstructure response is presented.

\section{Aerodynamic Solver}

\section{A. Governing Equations}

The governing equations solved are the three-dimensional, compressible Navier-Stokes equations. These equations are cast in strong conservative form introducing a general time-dependent curvilinear coordinate transformation $(x, y, z, t) \rightarrow(\xi, \eta, \zeta, \tau)$. In vector notation, and employing non-dimensional variables, the equations are:

$$
\frac{\partial}{\partial \tau}\left(\frac{\vec{U}}{J}\right)+\frac{\partial \hat{F}}{\partial \xi}+\frac{\partial \hat{G}}{\partial \eta}+\frac{\partial \hat{H}}{\partial \zeta}=\frac{1}{R e}\left[\frac{\partial \hat{F}_{v}}{\partial \xi}+\frac{\partial \hat{G}_{v}}{\partial \eta}+\frac{\partial \hat{H}_{v}}{\partial \zeta}\right]
$$

Here $\vec{U}=\{\rho, \rho u, \rho v, \rho w, \rho E\}$ denotes the solution vector and $J$ is the transformation Jacobian. The inviscid and viscous fluxes, $\hat{F}, \hat{G}, \hat{H}, \hat{F}_{v}, \hat{G}_{v}, \hat{H}_{v}$ can be found, for instance, in Ref. 11 . In the expressions above, $u, v, w$ are the Cartesian velocity components, $\rho$ the density, $p$ the pressure, and $T$ the temperature. All flow variables have been normalized by their respective freestream values except for pressure which has been nondimensionalized by $\rho_{\infty} u_{\infty}^{2}$. The system of equations is closed using the perfect gas law $p=\rho T / \gamma M_{\infty}^{2}$, Sutherland's formula for viscosity, and the assumption of a constant Prandtl number, $\operatorname{Pr}=0.72$.

\section{B. Spatial Discretization}

A finite-difference approach is employed to discretize the flow equations. For any scalar quantity, $\phi$, such as a metric, flux component or flow variable, the spatial derivative $\phi^{\prime}$ along a coordinate line in the transformed plane is obtained by solving the tridiagonal system:

$$
\alpha \phi_{i-1}^{\prime}+\phi_{i}^{\prime}+\alpha \phi_{i+1}^{\prime}=b \frac{\phi_{i+2}-\phi_{i-2}}{4}+a \frac{\phi_{i+1}-\phi_{i-1}}{2}
$$

where $\alpha=\frac{1}{3}, a=\frac{14}{9}$ and $b=\frac{1}{9}$. This choice of coefficients yields at interior points the compact five-point, sixth-order algorithm of Lele. ${ }^{12}$ At boundary points 1, 2, IL-1 and $I L$, fourth- and fifth-order one-sided formulas are utilized which retain the tridiagonal form of the interior scheme. ${ }^{13,14}$

Compact-difference discretizations, like other centered schemes, are non-dissipative and are therefore susceptible to numerical instabilities due to the growth of spurious high-frequency modes. These difficulties originate from several sources including mesh non-uniformity, approximate boundary conditions and nonlinear flow features. In order to ensure long-term numerical stability, while retaining the improved accuracy of the spatial compact discretization, a high-order implicit filtering technique ${ }^{15,16}$ is incorporated. If a component of the solution vector is denoted by $\phi$, filtered values $\hat{\phi}$ are obtained by solving the tridiagonal system,

$$
\alpha_{f} \hat{\phi}_{i-1}+\hat{\phi}_{i}+\alpha_{f} \hat{\phi}_{i+1}=\Sigma_{n=0}^{N} \frac{a_{n}}{2}\left(\phi_{i+n}+\phi_{i-n}\right)
$$

Equation 3 is based on templates proposed in Refs. 12 and 17, and with proper choice of coefficients, provides a $2 N$ th-order formula on a $2 N+1$ point stencil. The coefficients, $a_{0}, a_{1}, \ldots a_{N}$, derived in terms of the single parameter $\alpha_{f}$ using Taylor- and Fourier-series analysis, are given in Ref. 13, along with detailed spectral filter responses. In the present study, an eighth-order filter operator with $\alpha_{f}=0.3$ is applied at 
interior points. For near-boundary points, the filtering strategies described in Refs. 16 and 18 are employed. Filtering is applied to the conserved variables, and sequentially in each coordinate direction.

\section{Time Integration}

For wall-bounded viscous flows, the stability constraint of explicit time-marching schemes is too restrictive and the use of an implicit approach becomes necessary. For this purpose, the implicit approximately-factored scheme of Beam and Warming ${ }^{19}$ is incorporated and augmented through the use of Newton-like subiterations in order to achieve second-order temporal and sixth-order spatial accuracy. In delta form, the scheme may be written as

$$
\begin{aligned}
& {\left[J^{-1^{p+1}}+\phi^{i} \Delta \tau \delta_{\xi}^{(2)}\left(\frac{\partial \hat{F}^{p}}{\partial U}-\frac{1}{R e} \frac{\partial \hat{F}_{v}^{p}}{\partial U}\right)\right] J^{p+1} \times\left[J^{-1^{p+1}}+\phi^{i} \Delta \tau \delta_{\eta}^{(2)}\left(\frac{\partial \hat{G}^{p}}{\partial U}-\frac{1}{R e} \frac{\partial \hat{G}_{v}^{p}}{\partial U}\right)\right] J^{p+1} \times} \\
& {\left[J^{-1^{p+1}}+\phi^{i} \Delta \tau \delta_{\zeta}^{(2)}\left(\frac{\partial \hat{H}^{p}}{\partial U}-\frac{1}{R e} \frac{\partial \hat{H}_{v}^{p}}{\partial U}\right)\right] \Delta U=-\phi^{i} \Delta \tau\left[J^{-1^{p+1}} \frac{(1+\phi) U^{p}-(1+2 \phi) U^{n}+\phi U^{n-1}}{\Delta \tau}\right.} \\
& \left.+U^{p}(1 / J)_{\tau}^{p}+\delta_{\xi}\left(\hat{F}^{p}-\frac{1}{R e} \hat{F}_{v}^{p}\right)+\delta_{\eta}\left(\hat{G}^{p}-\frac{1}{R e} \hat{G}_{v}^{p}\right)+\delta_{\zeta}\left(\hat{H}^{p}-\frac{1}{R e} \hat{H}_{v}^{p}\right)\right]
\end{aligned}
$$

where

$$
\phi^{i}=\frac{1}{1+\phi}, \quad \Delta U=U^{p+1}-U^{p} .
$$

For the first subiteration, $p=1, U^{p}=U^{n}$ and as $p \rightarrow \infty, U^{p} \rightarrow U^{n+1}$. The spatial derivatives in the implicit (left-hand-side) operators are represented using standard second-order centered approximations whereas high-order discretizations are employed for the explicit terms (right-hand side). Although not shown in Eqn. 4, nonlinear artificial dissipation terms ${ }^{20,21}$ are appended to the implicit operator to enhance stability. In addition, for improved efficiency, the approximately-factored scheme is recast in diagonalized form. ${ }^{22}$ Any degradation in solution accuracy caused by the second-order implicit operators, artificial dissipation and the diagonal form are eliminated through the use of subiterations. Typically, three subiterations are applied per time step.

\section{Implicit Large Eddy Simulation Methodology}

The high-fidelity implicit large eddy simulation (ILES) method to be used in the present computations was first proposed and investigated by Visbal et al. ${ }^{23}$ The underlying idea behind the approach is to capture with high accuracy the resolved part of the turbulent scales while providing for a smooth regularization procedure to dissipate energy at the represented but poorly resolved high wavenumbers of the mesh. In the present computational procedure the 6th-order compact difference scheme provides the high accuracy while the low-pass spatial filters provide the regularization of the unresolved scales. All this is accomplished with no additional sub-grid scale models as in traditional LES approaches. An attractive feature of this filtering ILES approach is that the governing equations and numerical procedure remain the same in all regions of the flow. In addition, the ILES method requires approximately half the computational resources of a standard dynamic Smagorinsky sub-grid scale LES model. This results in a scheme capable of capturing with highorder accuracy the resolved part of the turbulent scales in an extremely efficient and flexible manner. The ILES solver is embedded in a high-order overset-grid scheme which is utilized to provide flexibility for modeling complex geometries. It also serves as a domain decomposition mechanism for application of the high-order approach on massively-parallel, high-performance computing platforms.

\section{E. Boundary Conditions}

The boundary conditions for the flow domain are prescribed as follows. At the solid surface, the no slip condition is applied, requiring that the fluid velocity at the wing surface match the surface velocity. In addition, the adiabatic wall condition, $\frac{\partial T}{\partial n}=0$, and the normal pressure gradient condition $\frac{\partial p}{\partial n}=0$ are specified. Along the O-grid cut spatial periodicity is imposed by means of a grid overlap region. The flow is assumed to be symmetric at the symmetry plane.

The treatment of the farfield boundaries is based on the approach proposed and evaluated previously in Ref. 24 for some acoustic benchmark problems. This method exploits the properties of the high-order, low-pass filter in conjunction with a rapidly stretched mesh. As grid spacing increases away from the region of interest, energy not supported by the stretched mesh is reflected in the form of high-frequency modes 
which are annihilated by the discriminating spatial filter operator. An effective "buffer" zone is therefore created using a few grid points in each coordinate direction to rapidly stretch to the farfield boundary. No further need for the explicit incorporation of complicated boundary conditions or modifications to the governing equations is then required. Freestream conditions are specified along the inflow portion of the farfield boundary, while simple extrapolation of all variables is used on the outflow portion of the boundary. Freestream conditions are also specified on the spanwise farfield boundary.

\section{Structural Dynamics Solver (UM/NLABS)}

The geometrically-nonlinear structural dynamics solution is based on an asymptotic approach to the equations governing the dynamics of a general 3 -D anisotropic slender solid. ${ }^{25,26}$ It is implemented in the University of Michigan's Nonlinear Active Beam Solver (UM/NLABS) computer code. Assuming the presence of a small parameter (the inverse of the wing aspect ratio) allows for a multi-scale solution process, in which the problem is decomposed into separate cross-sectional (small-scale) and longitudinal (long-scale) analyses. The longitudinal problem solves for average measures of deformation of the reference line under given external excitations. The cross-sectional problem solves the local deformation for given values of the long-scale variables. Both problems are tightly coupled and together provide an efficient approximation to the displacement field in the original 3-D domain. A flow diagram of the process is shown in Figure 1.

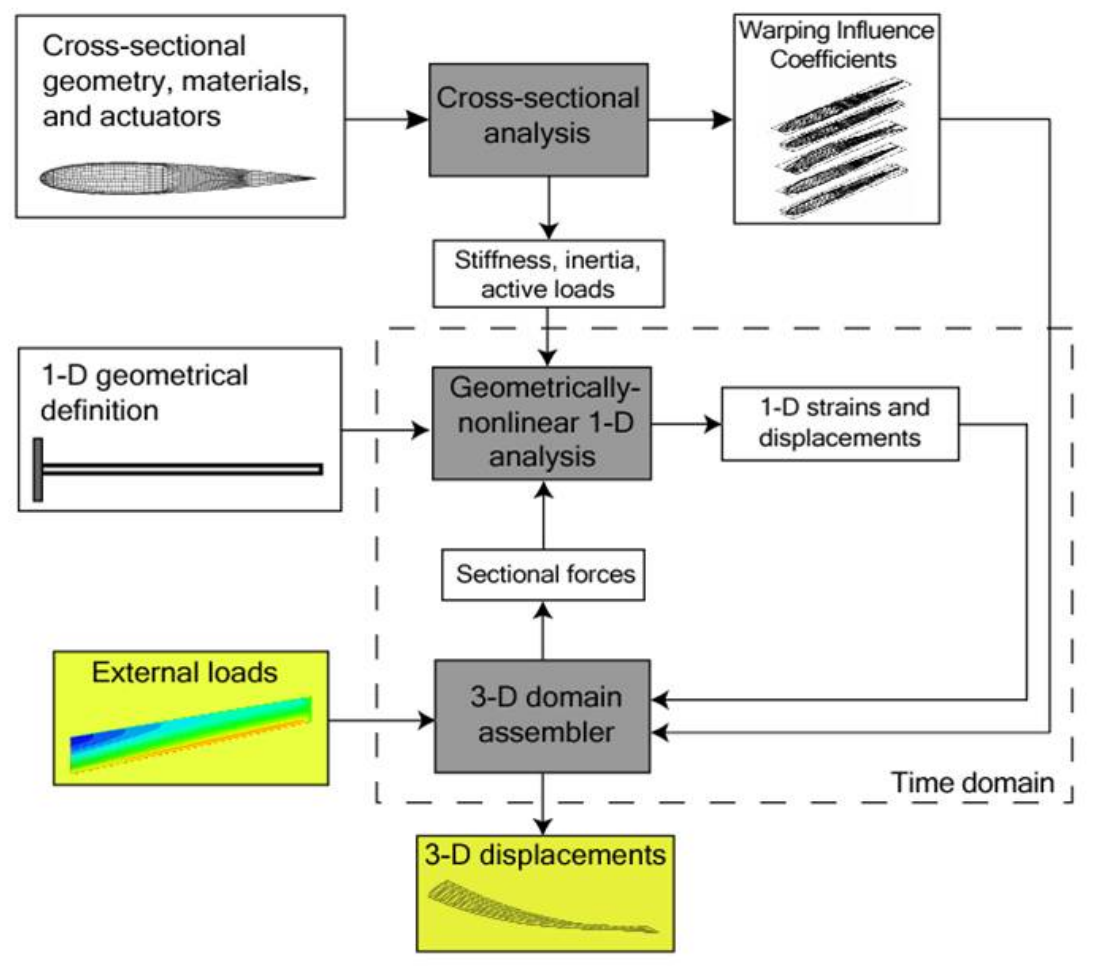

Figure 1. Asymptotic solution process for 3-D slender structures implemented in UM/NLABS

The structural formulation follows the variational-asymptotic method for the analysis of composite beams. ${ }^{27}$ The equations of motion for a slender anisotropic elastic 3-D solid are approximated by the recursive solution of a linear 2-D problem at each cross section, ${ }^{26}$ and a 1-D geometrically-nonlinear problem along the reference line. ${ }^{25}$ This procedure allows the asymptotic approximation of the 3-D warping field in the beam cross sections, which are used with the 1-D beam solution to recover a 3-D displacement field. The warping was approximated for the elastic degrees of freedom of a Timoshenko-beam model (extension and transverse shear, $\gamma$, and twist, bending about two directions, $\kappa$ ) and augmented with an arbitrary set of functions approximating the sectional deformation field (amplitude, $q$, and its derivative along the spanwise 
direction, $q^{\prime}$. These capture "non-classical" deformations, which are referred to as finite-section modes. And these new deformation modes are not restricted to be as small as the fundamental warping field. The solution of a variational problem yields the warping field corresponding to 1 -D beam strains $\left\{\gamma, \kappa, q, q^{\prime}\right\}$. In its first order approximation, it can be written as [26]

$$
w\left(x_{1}, x_{2}, x_{3}\right)=w_{\gamma}\left(x_{2}, x_{3}\right) \gamma\left(x_{1}\right)+w_{\kappa}\left(x_{2}, x_{3}\right) \kappa\left(x_{1}\right)+w_{q_{n}}\left(x_{2}, x_{3}\right) q_{n}\left(x_{1}\right)+w_{q_{n}^{\prime}}\left(x_{2}, x_{3}\right) q_{n}^{\prime}\left(x_{1}\right)+H O T
$$

where $\left\{\begin{array}{llll}w_{\gamma} & w_{\kappa} & w_{q_{n}} & w_{q_{n}^{\prime}}\end{array}\right\}$ are the first-order warping influence coefficients. Using this approximation for the warping field, the cross-section problem gives the strain energy per unit length of the beam:

$$
v=\frac{1}{2}\left\{\begin{array}{llll}
\gamma^{T} & \kappa^{T} & q^{T} & q^{\prime T}
\end{array}\right\}[S]\left\{\begin{array}{c}
\gamma \\
\kappa \\
q \\
q^{\prime}
\end{array}\right\}+H O T
$$

Here, the constant matrix $[S]$ is the first-order asymptotic approximation to the stiffness matrix. The integration of the kinetic energy can be directly done as function of the 1-D variables, yielding:

$$
K E=\frac{1}{2}\left\{\begin{array}{ccc}
V_{B}^{T} & \Omega_{B}^{T} & \dot{q}_{n}^{T}
\end{array}\right\}[M]\left\{\begin{array}{c}
V_{B} \\
\Omega_{B} \\
\dot{q}_{n}
\end{array}\right\}
$$

where the constant matrix $[M]$ is the inertia matrix for the cross section. From the resulting 1-D problem, the geometrically-nonlinear dynamic equations of equilibrium along the reference line (as presented in Ref. 25) are written as

$$
\begin{aligned}
\left(\frac{d}{d t}+\tilde{\Omega}_{B}\right) P_{B} & =\left(\frac{d}{d x}+\tilde{K}_{B}\right)\left(F_{B}-f_{1}\right)+f_{0} \\
\left(\frac{d}{d t}+\tilde{\Omega}_{B}\right) H_{B}+\tilde{V}_{B} P_{B} & =\left(\frac{d}{d x}+\tilde{K}_{B}\right)\left(M_{B}-m_{1}\right)+\left(\tilde{e}_{1}+\tilde{\gamma}\right) F_{B}+m_{0} \\
\frac{d}{d t} Q_{t} & =\frac{d}{d x}\left(Q_{s_{1}}-f_{s_{1}}\right)-\left(Q_{s_{0}}-f_{s_{0}}\right) .
\end{aligned}
$$

where the generalized forces and momenta are all expressed in their components in a reference frame attached to the deformed beam reference line. The first two equations imply equilibrium of forces and moments. The last equation in Eqns. 9 includes the set of equilibrium equations corresponding to the finite-section modes. With the warping influence coefficients given by Eq. 6, the applied forces per unit length in Eq. 9 are

$$
\begin{aligned}
f_{0} & =\int_{A(x)} \mu_{B} d A \\
f_{s_{0}} & =\int_{A(x)}\left(\Psi_{q}^{T}+w_{q}^{T}\right) \mu_{B} d A \\
m_{0} & =\int_{A(x)} \xi_{B} \mu_{B} d A \\
f_{1} & =\int_{A(x)} w_{\gamma}^{T} \mu_{B} d A \\
f_{s_{1}} & =\int_{A(x)} w_{q^{\prime}}^{T} \mu_{B} d A \\
m_{1} & =\int_{A(x)} w_{\kappa}^{T} \mu_{B} d A
\end{aligned}
$$

The present implementation of this formulation follows the approach described in Ref. 25, where the solution to Eq. 9 is done by means of a finite-element discretization of a mixed-variational form of the equations. Therefore, although they are analyzed independently, the small and long-scale problems are intimately linked in the detailed approximation to the solution. This is particularly important in the generation of the solid side of an aeroelastic model: the interface of the structural model consists of the actual wetted surfaces of the vehicle, without extrapolations from the motion of a reduced-dimension structural model, nor the assumption of rigid cross sections required by beam theories. 


\section{Aerodynamic/Structural Coupling}

Coupling of the aerodynamics with the wing structural response occurs through the imposed aerodynamic loads and the resulting structural deflection of the wing, which is returned to the aerodynamic grid. Communication between the noncoincident aerodynamic and structural surface meshes is accomplished using a local bilinear interpolation procedure ${ }^{28}$ for the physical quantities to be passed between the aerodynamic and structural solvers. Implicit coupling of these two sets of equations is achieved by a global subiteration strategy. During each subiteration the aerodynamic forces are updated in the structural solver and the new surface displacements are provided to the aerodynamic solver. Using this approach the temporal lag between the aerodynamic and structural equations may be eliminated and a complete synchronization of the aerodynamic/structural equation set is achieved. Any factorization or linearization errors introduced in the equations may also be eliminated using this global subiteration procedure. The resulting coupled procedure retains second order temporal accuracy.

When solving fluid/structure interactions, the aerodynamic mesh must be allowed to move in accordance with the motion of the structural surface. A simple algebraic method described in Ref. 29 deforms the aerodynamic mesh to accommodate the wing motion. This grid motion strategy has proved adequate for the wing motions considered in the present work.

\section{Results}

The problem to be addressed is the simulation of a three-dimensional rectangular wing with a uniform NACA0012 cross section oscillating in water in pure heave. Water tunnel studies have been performed by Heathcote et al. ${ }^{4}$ to study the effect of spanwise flexibility on the thrust, lift, and propulsive efficiency for this configuration. A schematic of the experimental setup is shown in Figure 2a. Three wings of 0.3-m span

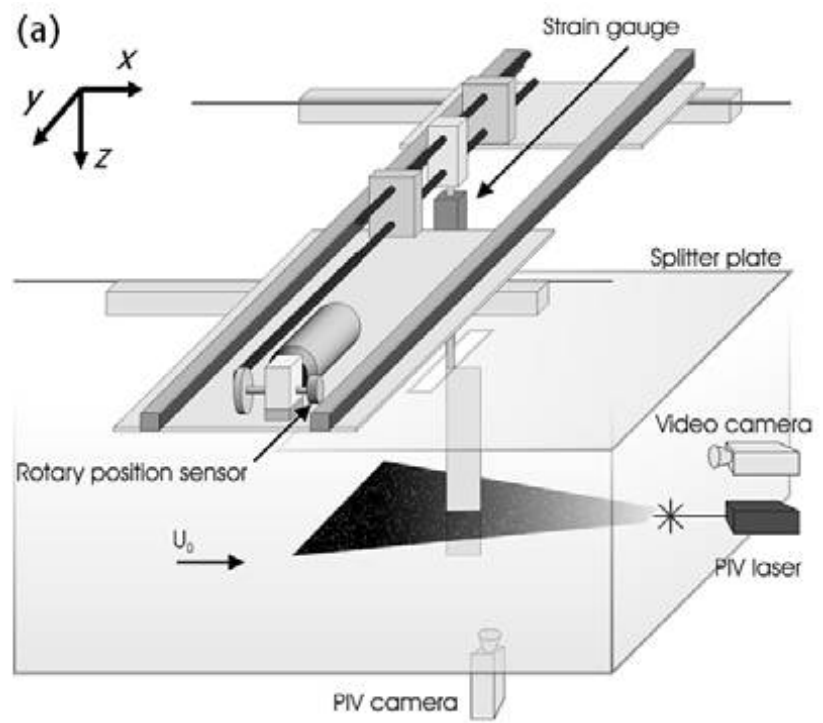

(b)
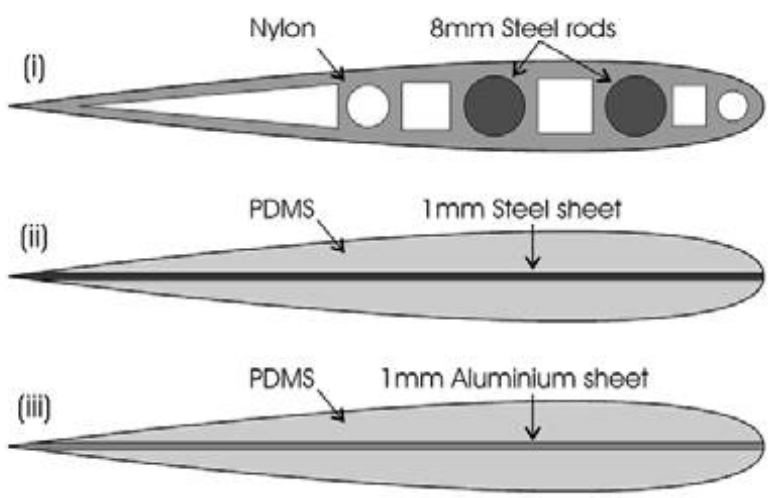

Figure 2. Experimental setup and wing cross-section. ${ }^{4}$ (a) Water-tunnel experimental setup (b) Inflexible (i), Flexible (ii), and Highly flexible (iii) wing cross-sections

and 0.1-m chord with increasing flexibility were constructed, Fig. 2b. The leading edge at the wing root was actuated by a prescribed oscillatory plunging motion, $z=z_{\text {root }} \sin (2 k t)$, Figure 3 , where $z_{\text {root }}$ is the non-dimensional heave amplitude and $k=\frac{\omega c}{2 U_{\infty}}$ is the reduced frequency. The cases computed in the present work specified the following values for these parameters: $k=1.82$, period $T=1.726, z_{\text {root }}=0.175$ and Reynolds number, $R e=3.0 \times 10^{4}$.

A grid system of 13 overset meshes has been developed around the NACA0012 wing for the fluid dynamic computations. The main grid for the wing is shown in Fig. 4 where every other mesh point has been removed for clarity. The main grid consist of $503 \times 355 \times 229$ mesh points in the circumferential, spanwise and normal directions respectively. The maximum spacing in the chordwise direction is $\Delta x=0.0075$ and the minimum 


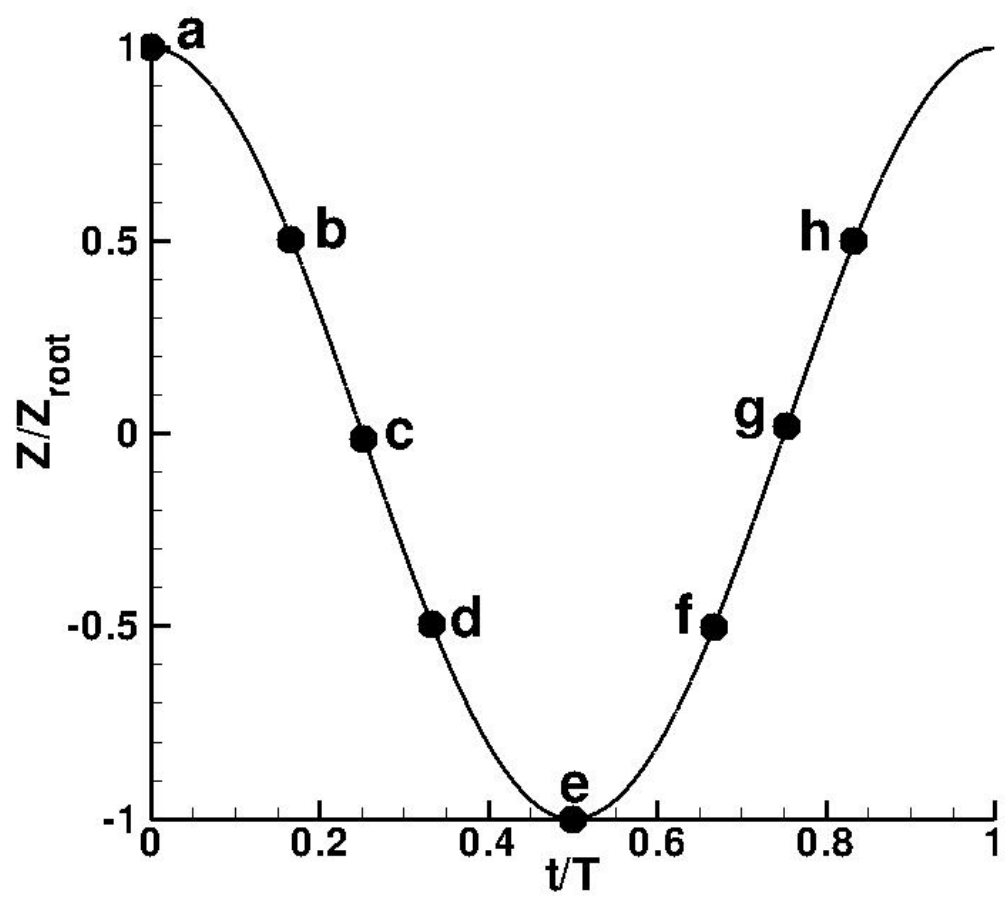

Figure 3. Wing Root Motion

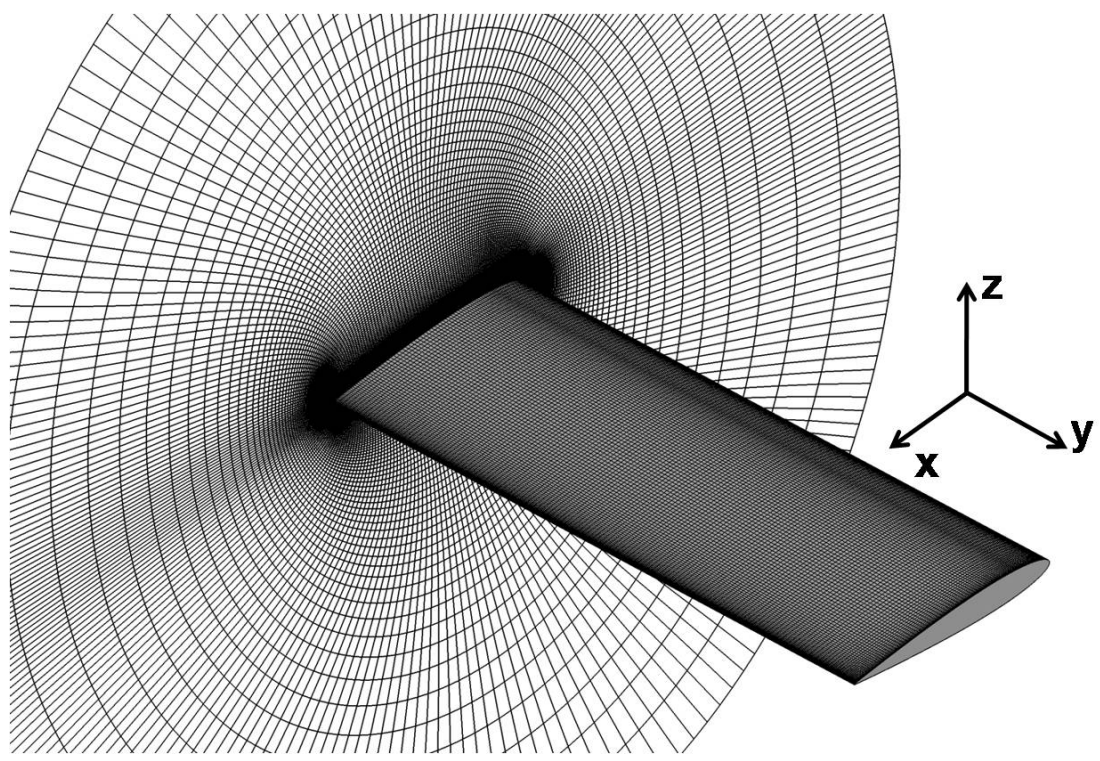

Figure 4. Fluid dynamics grid for NACA0012 wing 
spacing at the wall is $\Delta z=0.0001$. In the spanwise direction a uniform spacing $\Delta y=0.01$ is specified over the majority of the wing with the grid spacing being reduced to $\Delta y=0.001$ at the wing tip. The main wing grid extends a quarter chord past the wing tip and 2.6 chord lengths away from the airfoil. An additional 12 overset meshes have been developed to resolve the blunt wing tip region and to extend the mesh to the farfield boundaries. These meshes are progressively coarsened in the farfield where less mesh resolution is required. The overall mesh system extends 100 chord lengths in the body normal direction and 50 chord lengths in the spanwise direction. This overset mesh system was further decomposed into 497 subdomains which were each assigned to individual processors for parallel processing.

Computations for the rigid, flexible and highly flexible wing structures in Heathcote et al.'s experiment ${ }^{4}$ are performed. For the rigid wing the structure is considered to be infinitely stiff and no structural model is required. For the flexible and highly flexible wing cases the Flexible- 1 and Flexible- 3 models presented in Reference 7 are adopted. Chordwise deformation was reported as being negligible in the experiment, ${ }^{4}$ therefore, a beam model with six elastic degrees of freedom, corresponding to extension, twist, and shear and bending in two directions, was chosen. The beam reference line (cantilevered to a plunging frame of reference) is chosen along the leading edge of the wing. A beam finite-element discretization with 39 elements along the semi-span is used. 75 recovery nodes are employed on each cross section resulting in a structured grid of 3000 interface points which define the 3D structural solution. The structural properties for each case are assumed to be uniform throughout the semi-span and the cross-sectional properties are evaluated with respect to the leading edge point. Table 1 gives the geometric and mechanical properties for each wing model. The contribution of the PDMS rubber material to the overall mass and stiffness properties is assumed

Table 1. Geometric and Mechanical Properties of the Wing Models

\begin{tabular}{cccc}
\hline \hline & Rigid & Flexible & Highly Flexible \\
\hline Semi-Span $[\mathrm{m}]$ & 0.3 & 0.3 & 0.3 \\
Chord Length $[\mathrm{m}]$ & 0.1 & 0.1 & 0.1 \\
Plate Thickness $[\mathrm{m}]$ & 0.001 & 0.001 & 0.001 \\
Material Density $\left[\mathrm{kg} / \mathrm{m}^{3}\right]$ & - & 7800 & 2700 \\
Young's Modulus $[\mathrm{GPa}]$ & - & 210 & 40 \\
\hline \hline
\end{tabular}

to be negligible in each model. As discussed in Reference 7 a $43 \%$ drop in the value of Young's modulus over the standard value for aluminum was required for the highly flexible model to approximate the proper magnitude of the deflection encountered in the experiment. The need for this assumption and the impact of neglecting the mass of the PDMS rubber material are a source for further investigation that is beyond the scope of the present paper.

\section{A. Comparison with Experiment - Rigid Wing}

Heathcote et al. ${ }^{4}$ performed PIV measurements of the vortical structures in the wake of the plunging wing. Figure 5 compares the wake vortex structure for the computation, Fig. 5a, with the experimental PIV measurements, Fig. 5b. Good overall agreement is seen between the experiments and computations. The computation captures the small scale structures in the wing wake as well as the general character of the shed vortical structure. The strength of the shed vortex is somewhat weaker in the computations as the vortex has already convected into a region where the grid has started to stretch. This mesh was not specifically designed to capture wake features. A second comparison is made at the midpoint of the downstroke in Figure 6. The high-order fluid solver has captured well both in character and strength the fine scale vortical structures being shed from the trailing edge of the wing. The previously shed vortical structure that has convected further downstream is still present in both the computation and experiment. These results demonstrate the ability of the high-order computational solver to capture the fine details of this highly unsteady flowfield.

Figure 7 plots the time histories of the thrust coefficient for both the computation and the experiment. The experimental thrust coefficient exhibits an asymmetric response between the downstroke and the upstroke of the wing. The maximum thrust on the downstroke attains a slightly higher value while the minimum thrust has a lower value. The reason for this asymmetric thrust observed in the experiment is not discussed in Reference 4. The present computations as well as previous computations by Chimakurthi et al. ${ }^{6}$ did not show this behavior. The maximum computed thrust agrees well with the lower thrust coefficient peaks 


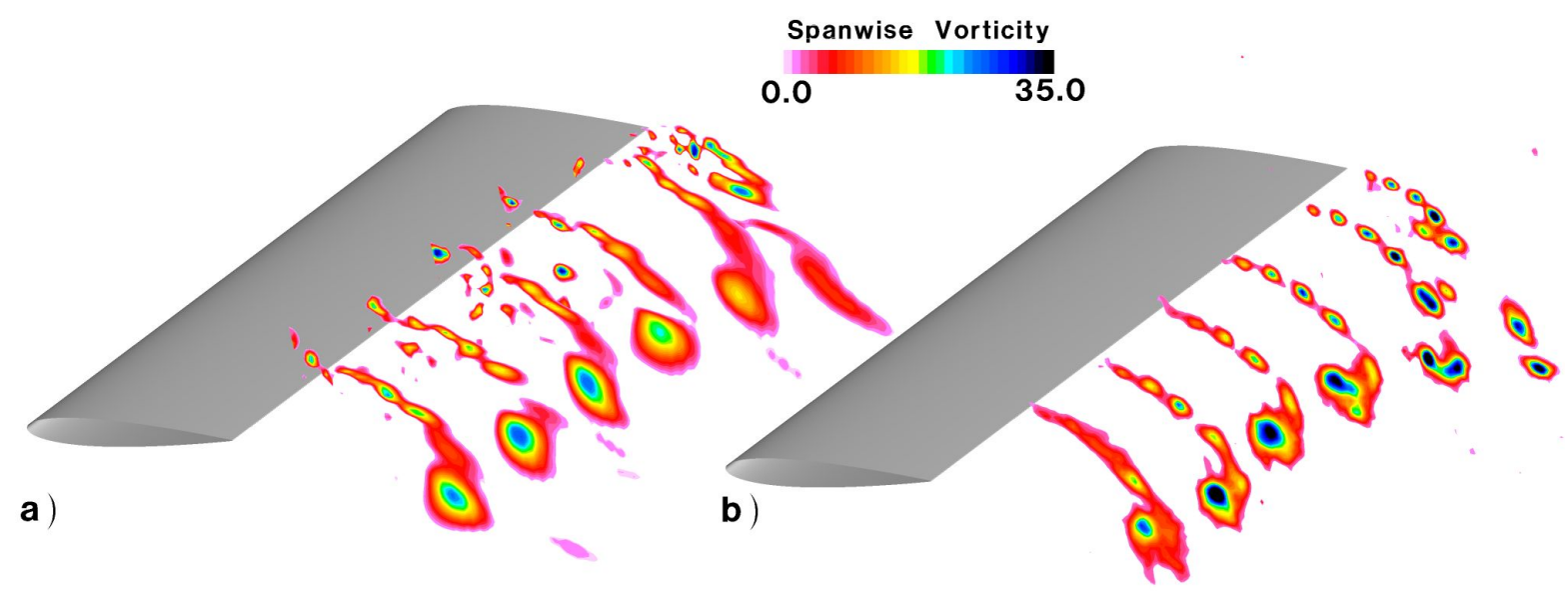

Figure 5. Comparison of wake structure at various spanwise stations at the peak of the upstroke, $t / T=0.0$ for the rigid wing: a) Computation, b) Experiment

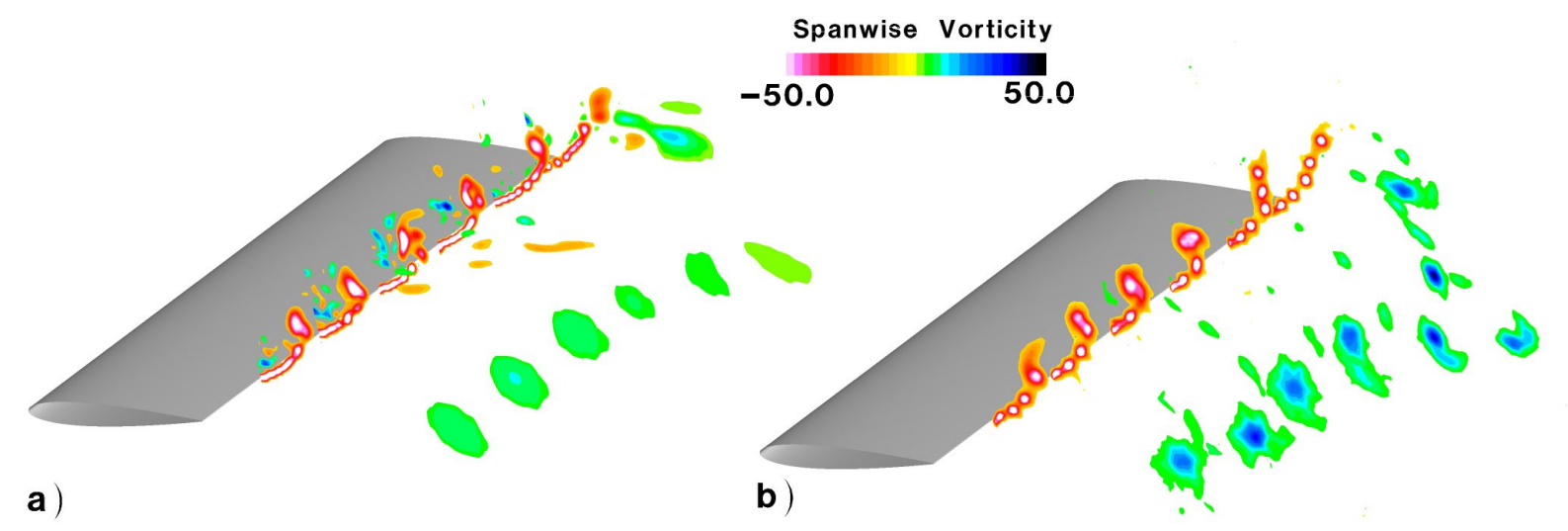

Figure 6. Comparison of wake structure at various spanwise stations at the midpoint of the downstroke, $t / T=0.25$ for the rigid wing: a) Computation, b) Experiment 


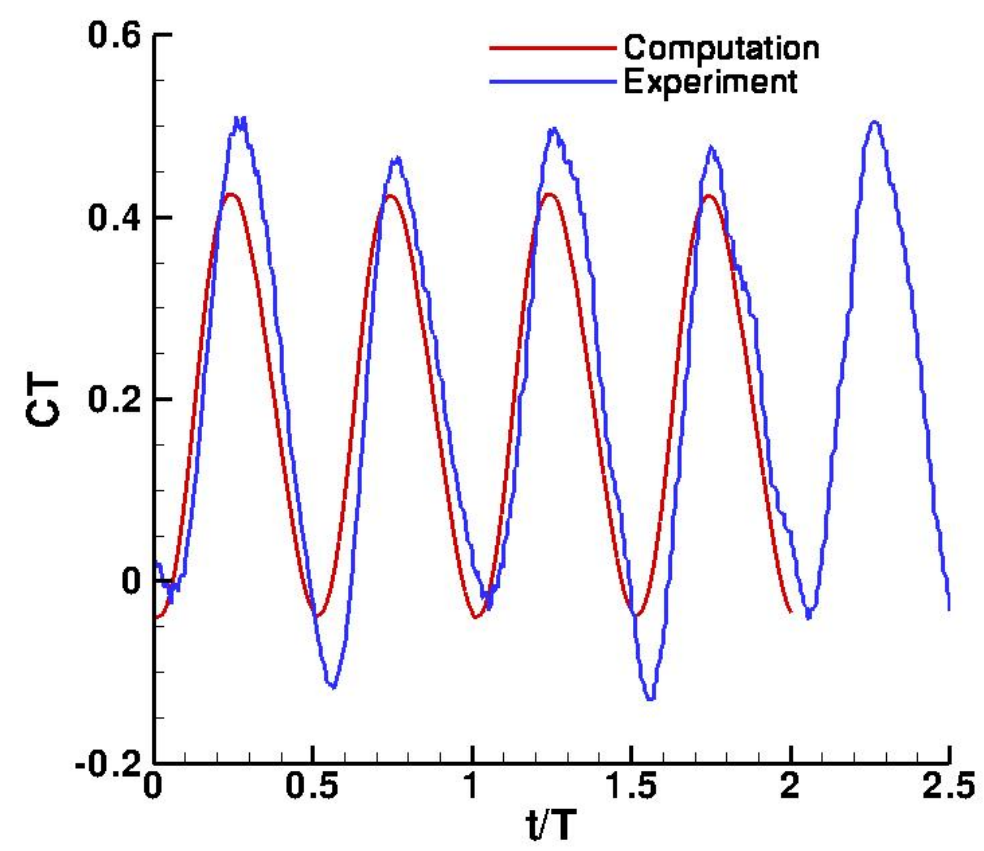

Figure 7. Comparison of the experimental and computational time histories of the thrust coefficient for the rigid wing

produced during the upstroke in the experiment. Good correlation between the mean thrust produced in the experiment and computation is also obtained, Table 2.

The computed lift coefficient time history for the rigid wing plunge case is compared with inviscid theory ${ }^{30}$ in Figure 8. Good agreement is seen between the inviscid theory and the current computations. This can be explained by the fact that for this high value of reduced frequency $(k=1.82)$ the magnitude of the lift coefficient is dominated by the acceleration of the airfoil (noncirculatory lift, Figure 8) which scales with $k^{2}$. The circulatory term primarily gives rise to the lag observed in the lift coefficient time history. Similar results have been shown by Visbal $^{9}$ for a plunging airfoil.

Table 2. Maximum Tip Displacement and Mean Thrust Data

\begin{tabular}{rrccc}
\hline \hline & & $Z_{\text {tip }} / Z_{\text {root }}$ & Phase Angle & $\overline{C T}$ \\
\hline Rigid & Experiment & & & 0.21 \\
& Computation & & & 0.195 \\
Flexible & Experiment & 1.64 & -25.1 & 0.32 \\
& Computation & 1.59 & -27.2 & 0.278 \\
Highly Flexible & Experiment & 1.76 & -117.0 & 0.11 \\
& Computation & 1.73 & -135.0 & 0.121 \\
\hline \hline
\end{tabular}

\section{B. Comparison with Experiment - Flexible Wing}

For the flexible wing case the time history of the computed wing tip deflection agrees well with the experimentally measured deflection, Figure 9. The maximum computational deflection matches within $3 \%$ the maximum experimental wing tip deflection, Table 2. A negative phase lag is observed between the imposed motion at the wing root and the motion of the wing tip with the experimental value being close to the computational value (see Table 2). 


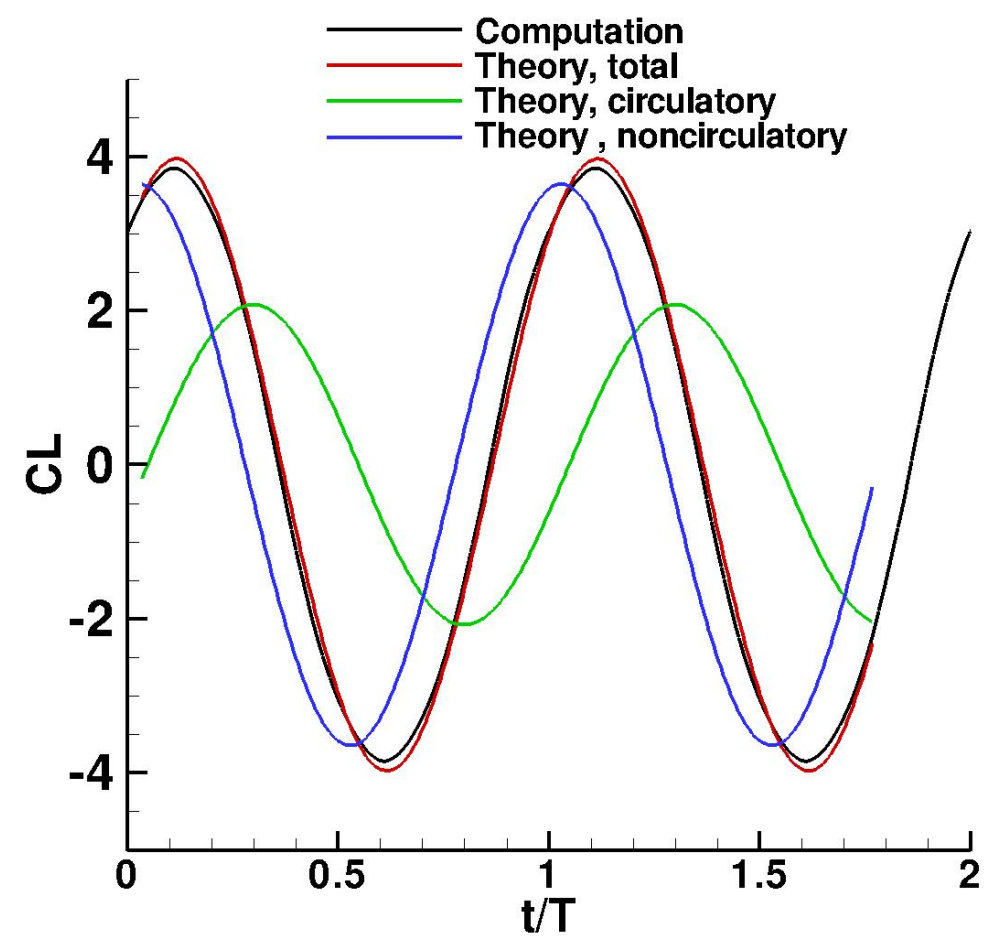

Figure 8. Comparison of the lift coefficient with theory ${ }^{30}$ for the rigid wing

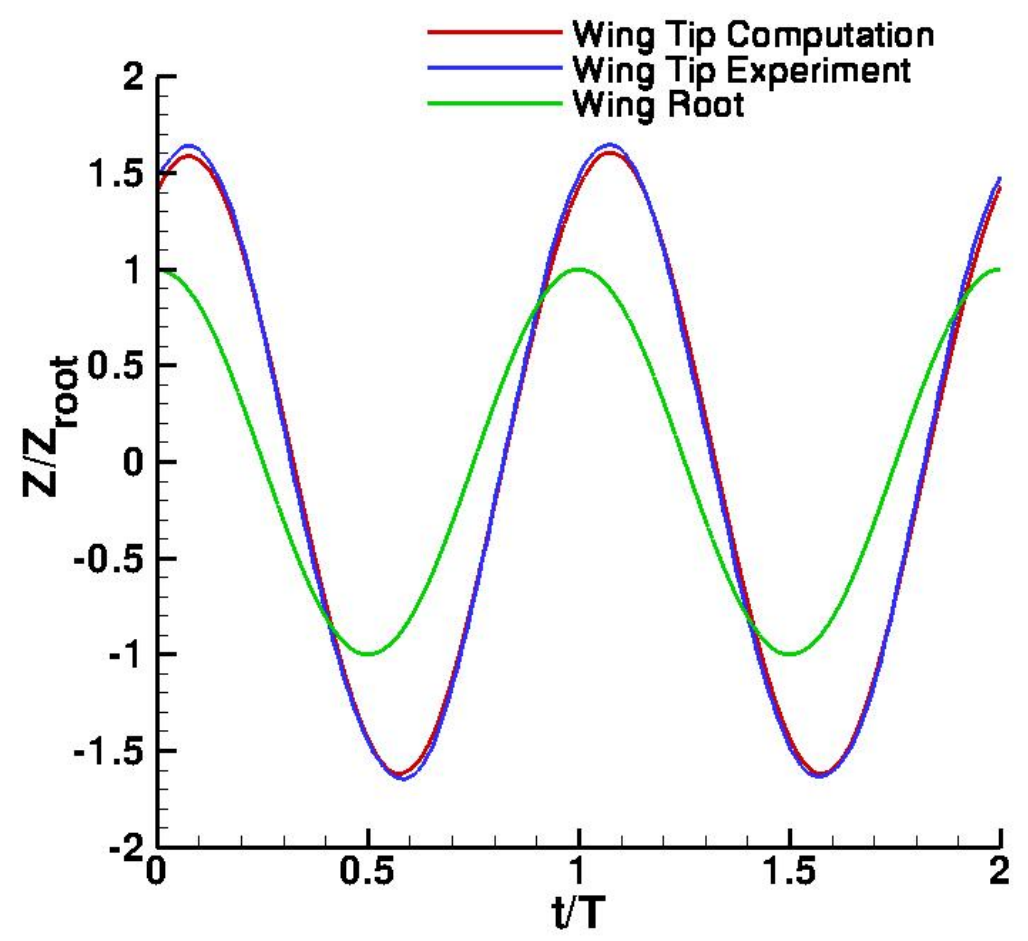

Figure 9. Comparison of the computed wing tip displacement with experiment for the flexible wing 
Figures 10 and 11 demonstrate the similarity between the computed and experimentally measured wake

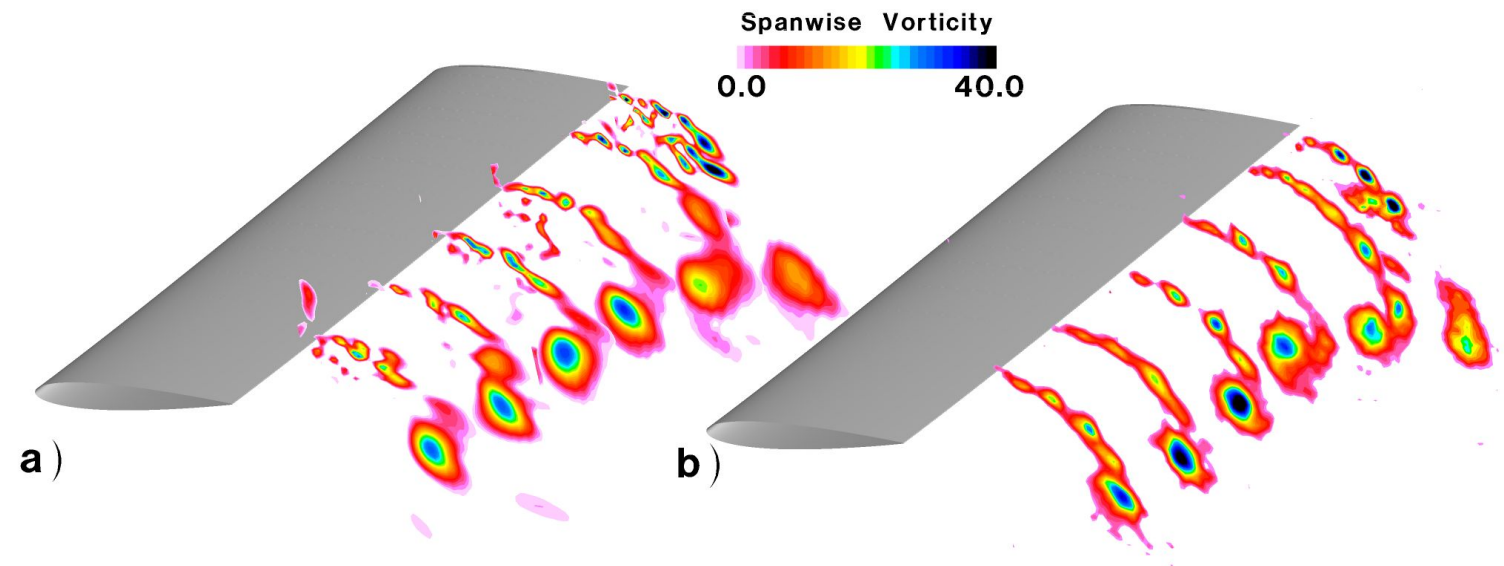

Figure 10. Comparison of wake structure at various spanwise stations at the peak of the upstroke, $t / T=0.0$ for the flexible wing: a) Computation, b) Experiment

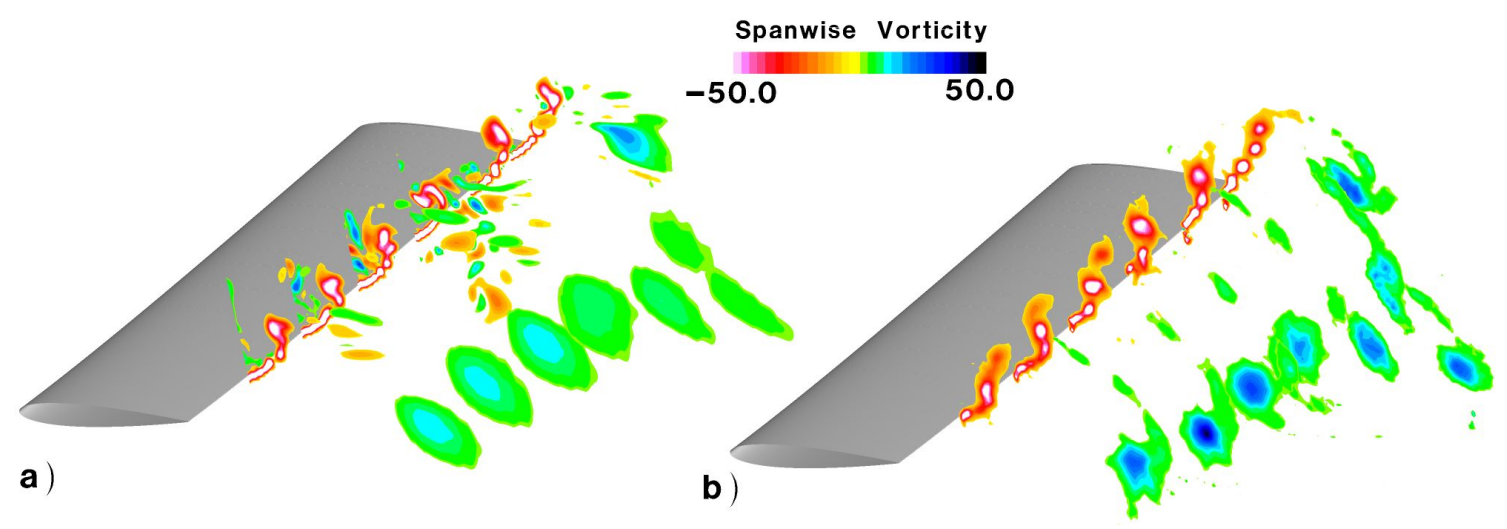

Figure 11. Comparison of wake structure at various spanwise stations at the midpoint of the downstroke, $\mathrm{t} / \mathrm{T}=\mathbf{0 . 2 5}$ for the flexible wing: a) Computation, b) Experiment

vortical structures as in the rigid case. At each time instant the computation has reproduced the general character of the shed vortical structure. In addition the fine scale vortical features in the separated shear layer are also replicated in the computations.

Figure 12 plots the experimental and computational thrust coefficient time histories. As in the rigid wing case, the experimental thrust coefficient shows larger peaks during the downstroke and smaller peaks during the upstroke of the plunging wing. This difference between the downstroke and upstroke is not observed in the computation which exhibits a more symmetric response between the downstroke and upstroke. The computations agree more closely with the thrust produced on the upstroke in the experiment though the maximum thrust produced is less. The computation also produces a smaller mean thrust than observed in the experiment, Table 2.

\section{Comparison with Experiment - Highly Flexible Wing}

For the highly flexible wing the time history of the computed wing tip deflection again aligns with the experimentally measured deflection, Figure 13. The maximum computational deflection matches within $1.7 \%$ the maximum experimental wing tip deflection, Table 2 . In this case a very large negative phase lag is observed both experimentally and computationally with the computation further lagging the experiment by $18^{\circ}$. As a result of this large phase lag the motion of the wing tip is out of phase with the motion of the wing 


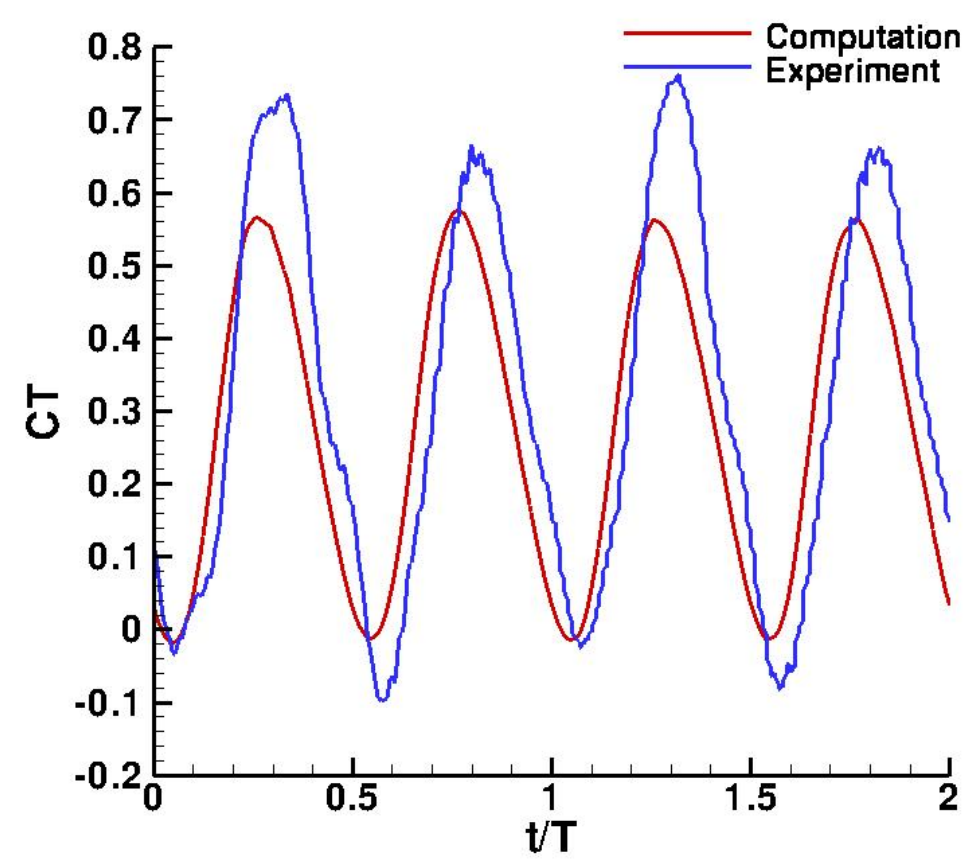

Figure 12. Comparison of the experimental and computational time histories of the thrust coefficient for the flexible wing

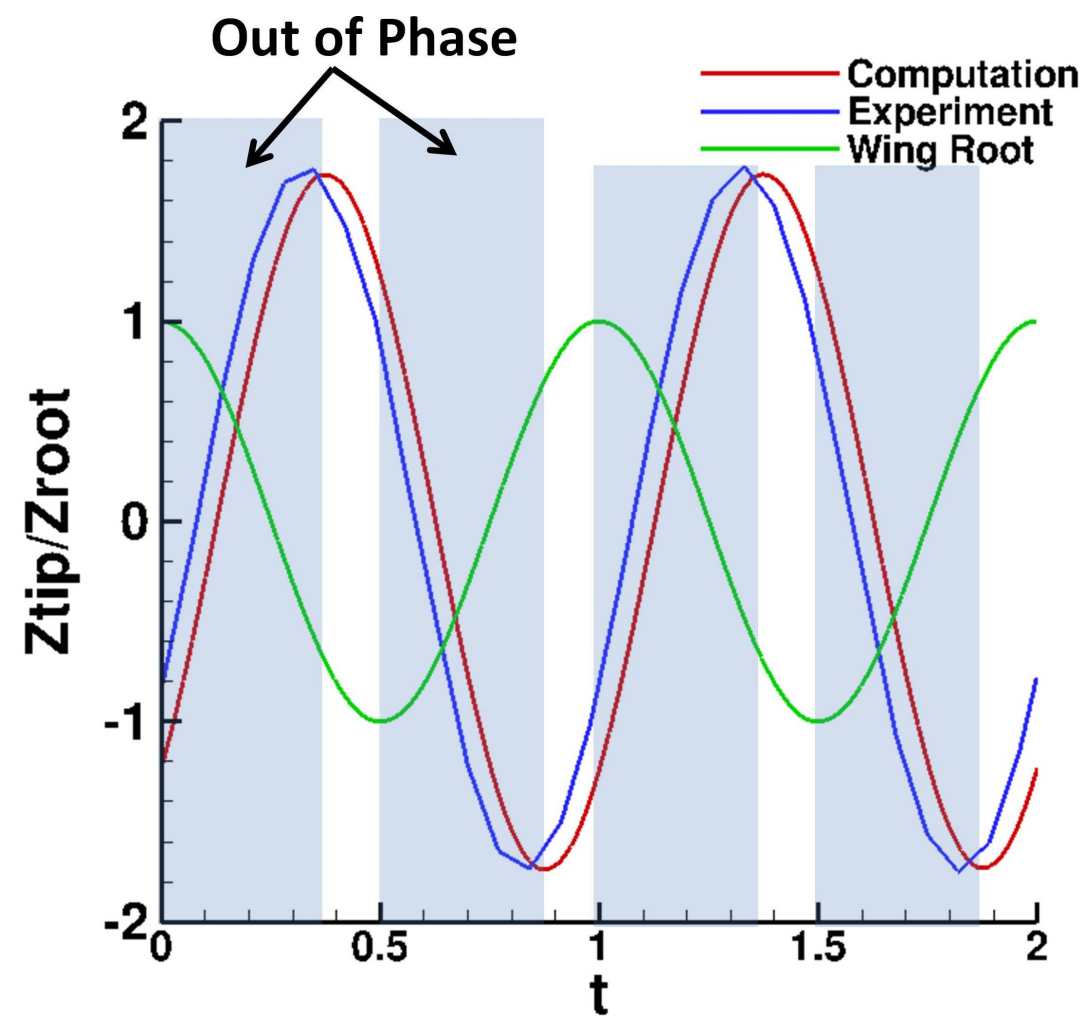

Figure 13. Comparison of the computed wing tip displacement with experiment for the highly-flexible wing 
root over substantial portions of the plunge cycle. The portions of the cycle where the wing tip and wing root are out of phase are highlighted by the blue boxes in Figure 13. The good qualitative and quantitative agreement between the experimental and computational structural response at the wing tip indicates that the current structural model produces a consistent response with the experiment despite the uncertainties associated with the modulus of elasticity and mass of the PDMS rubber material discussed previously.

Figures 14 and 15 demonstrate the good qualitative agreement between the computed and experimentally

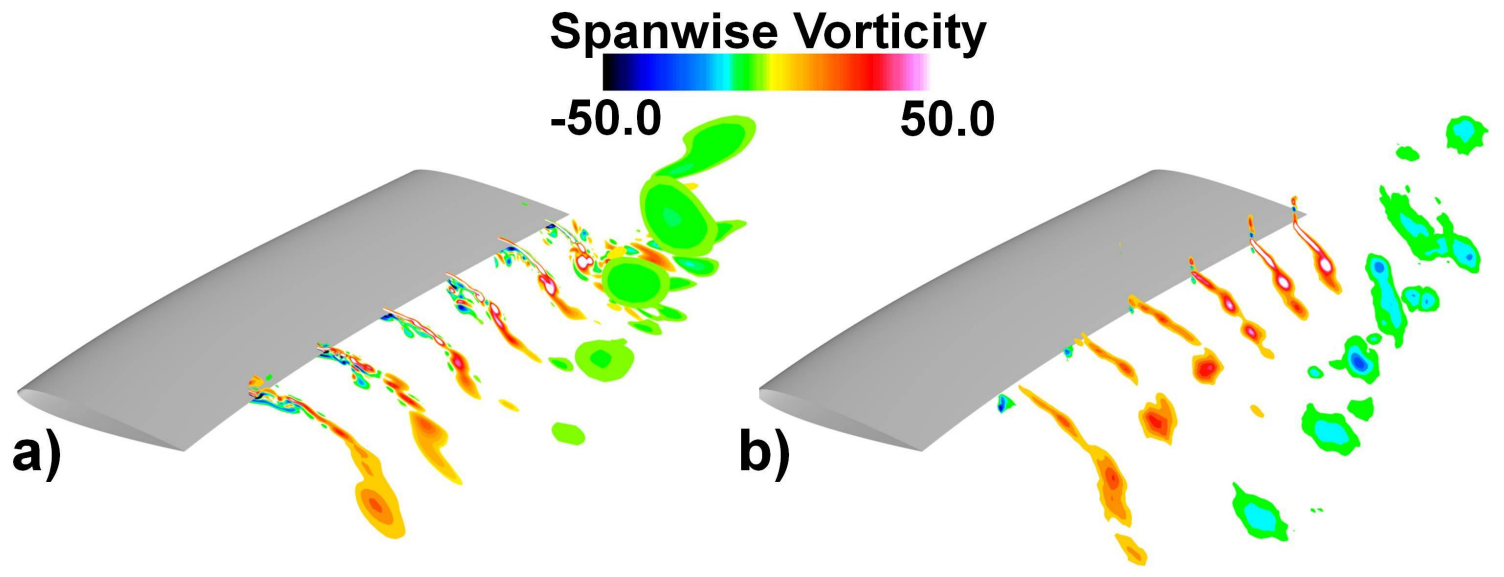

Figure 14. Comparison of wake structure at various spanwise stations at the peak of the upstroke, $t / T=0.0$ for the highly-flexible wing: a) Computation, b) Experiment

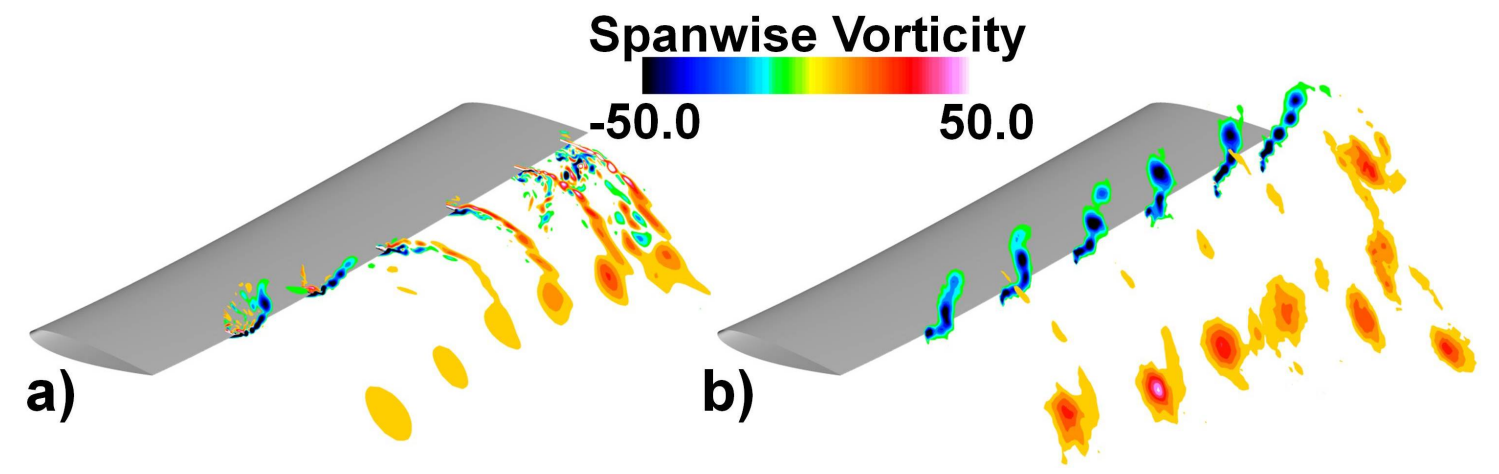

Figure 15. Comparison of wake structure at various spanwise stations at the midpoint of the downstroke, $\mathrm{t} / \mathrm{T}=\mathbf{0 . 2 5}$ for the highly flexible wing: a) Computation, b) Experiment

measured wake vortical structures as in the previous cases. At each time instant the computation has reproduced the general character of the shed vortical structure though some discrepancies are noted outboard on the wing at $t / T=0.25$, Figure 15 . This difference can be attributed in part to the phase shift between the structural deflections in the experiment and the computation. The fine scale vortical features in the separated shear layer noted in the experiment are again seen to be replicated in the computations.

The resulting experimental and computational thrust coefficient time histories are shown in Figure 16. While the magnitude of the mean thrust produced in the computations agrees with the experiment (see Table 2), there are distinct differences in the character of the results. The experimental thrust exhibits high frequency content not observed in the present or previous ${ }^{7}$ computational results. The source of this high frequency ringing is not clear. No evidence of a higher frequency in the structural response measurement, Figure 13, is noted. Furthermore, the flowfield measurements also do not indicate flow features that may be attributable to this high frequency content. The reason for the presence of the high frequency content in the experiment is a source for further investigation. 


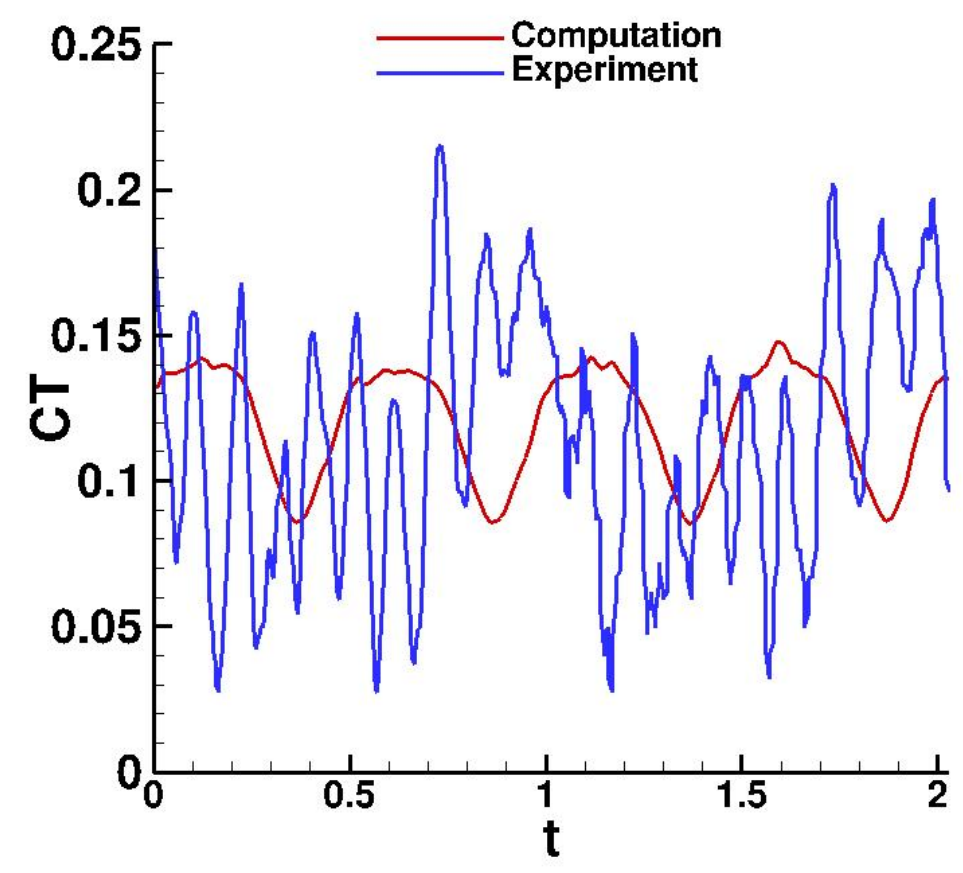

Figure 16. Comparison of the experimental and computational time histories of the thrust coefficient for the flexible wing

\section{Effect of Flexibility - Wing Flowfield}

\section{Flexible Case}

As was seen in Figure 9, the flexible wing exhibits a significant deflection at the wing tip due to the inherent flexibility of the structure and this deflection lags the imposed plunging motion at the wing root. This results in a spanwise variation in the deflection of the wing, Figure 17, as well as the development of a spanwise variation in the effective angle of attack, Figure 18, which results from the velocity of the wing surface, $\alpha_{e f f}=\tan ^{-1}\left(-\frac{d z}{d t} / U_{\infty}\right)$. Figure 19 demonstrates the impact of these effects on the global flowfield development on the upper surface of the wing. In this figure isosurfaces of vorticity magnitude are plotted with the isosurfaces being colored by the pressure coefficient. Figures $19 \mathrm{a}-\mathrm{h}$ correspond to the points a-h on the plunge cycle in Figure 3. Corresponding surface pressure coefficient contours on the upper surface are displayed in Figures $20 \mathrm{a}-\mathrm{h}$. Due to the relatively symmetric response between the downstroke and the upstroke of the wing in the computation an understanding of the flow on the under surface of the wing can be obtained by matching (a-d) for the topside with (e-h) for the bottomside in Figures 19 and 20 for the downstroke and viceversa for the upstroke.

At the top of the plunge cycle, Figure 19a, only minor differences between the rigid and flexible wing flowfields are observed. For the rigid wing distinct remnants of the previously shed leading edge vortex may still be seen near the trailing edge. For the flexible wing only dispersed small scale structures are seen except outboard on the wing where a concentrated region of small scale structures is observed. The presence of this feature will be discussed subsequently. The flexible wing exhibits higher pressure at the leading edge which results from the increasingly negative effective angle of attack towards the wing tip, Fig. 18.

During the downward plunging motion of the wing, the flowfield on the upper surface is characterized by the development of two features, the leading edge vortex and the tip vortex, Figures 19a-e. The rigid wing flowfield exhibits a fairly two-dimensional development except in the region very near the tip where the leading edge vortex is pinned to the wing tip leading edge. In contrast the flexible wing flowfield shows notable spanwise variation and distinct differences from the rigid wing. The leading edge vortex that develops increases in strength outboard on the wing due to the increase in effective angle of attack, Figure 18. This 


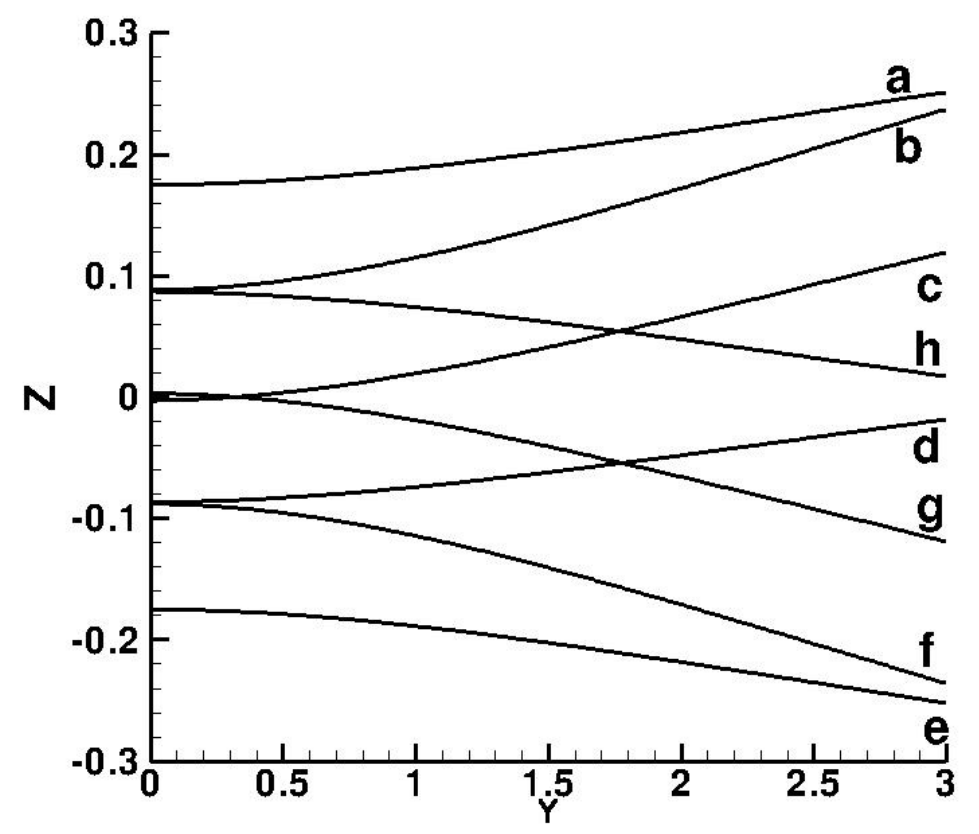

Figure 17. Spanwise deflection of the wing leading edge at points (a)-(h) in the plunge cycle, Fig. 3

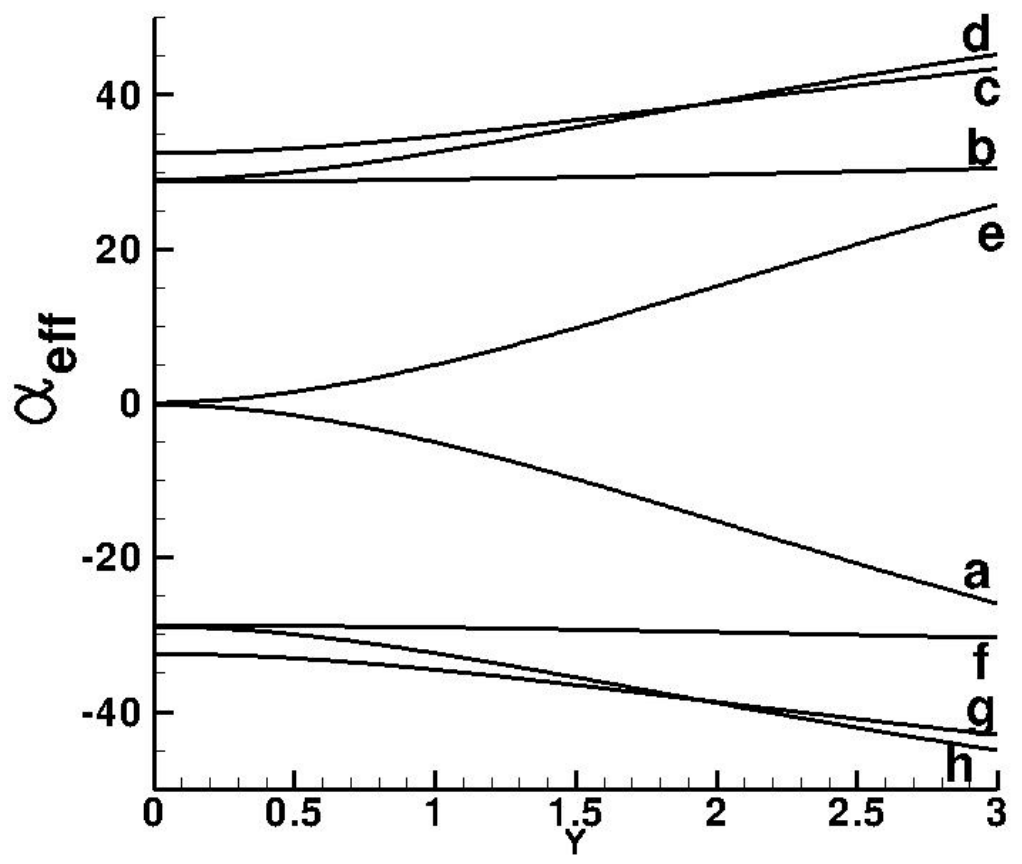

Figure 18. Spanwise variation of the effective angle of attack at points (a)-(h) in the plunge cycle, Fig. 3 


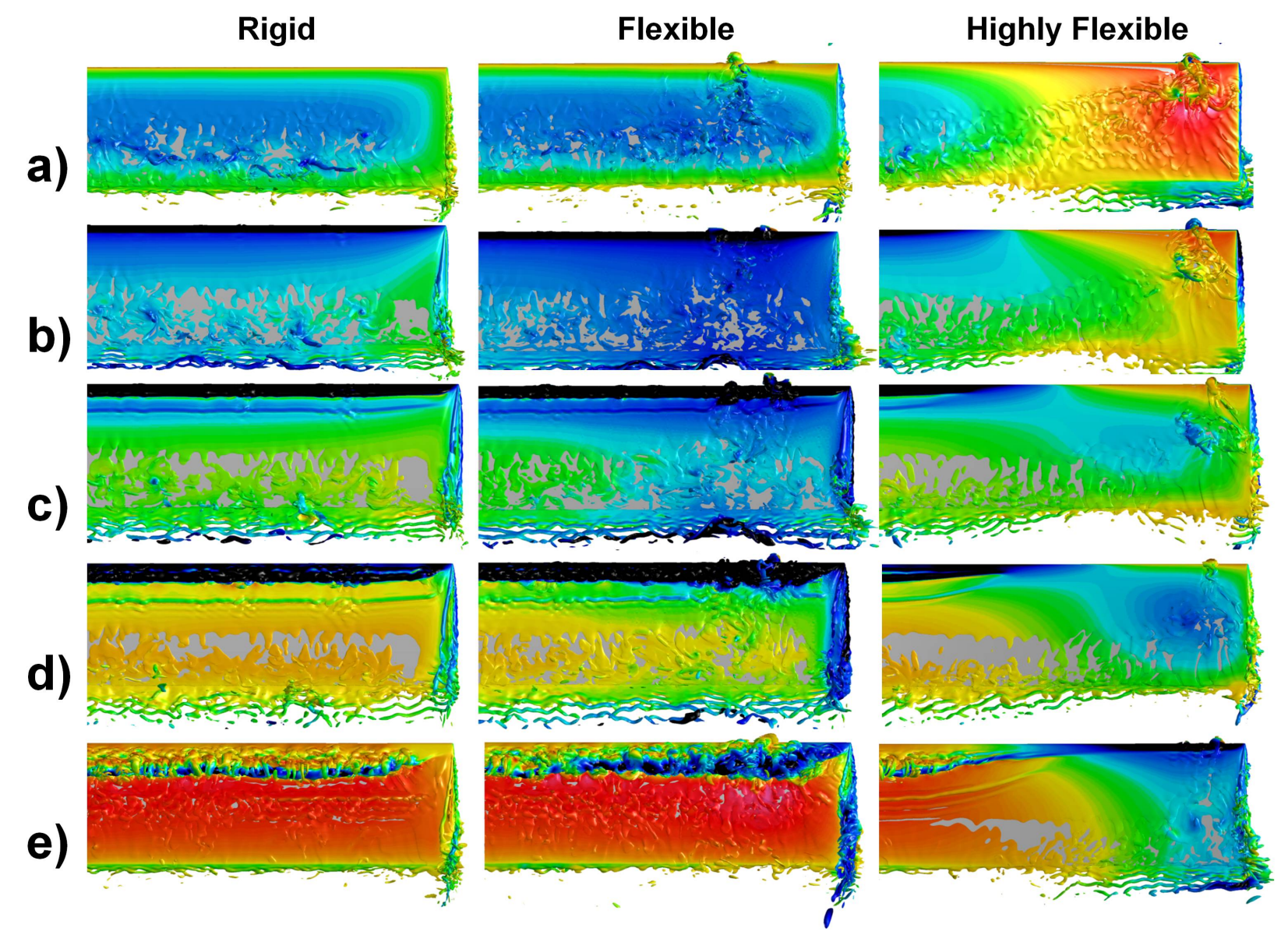

Figure 19. Comparison of Flow Structure (Isosurfaces of Vorticity Magnitude Colored by Pressure Coefficient) on the Wing Upper Surface for the Rigid, Flexible and Highly-Flexible Cases
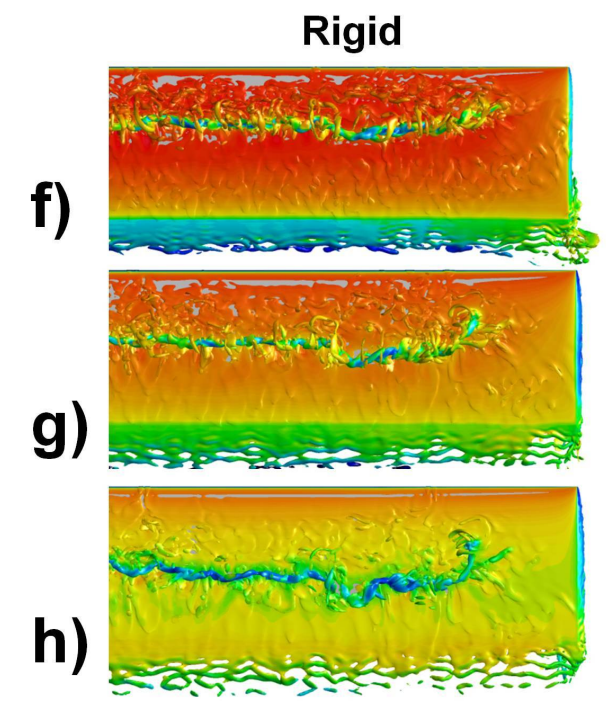
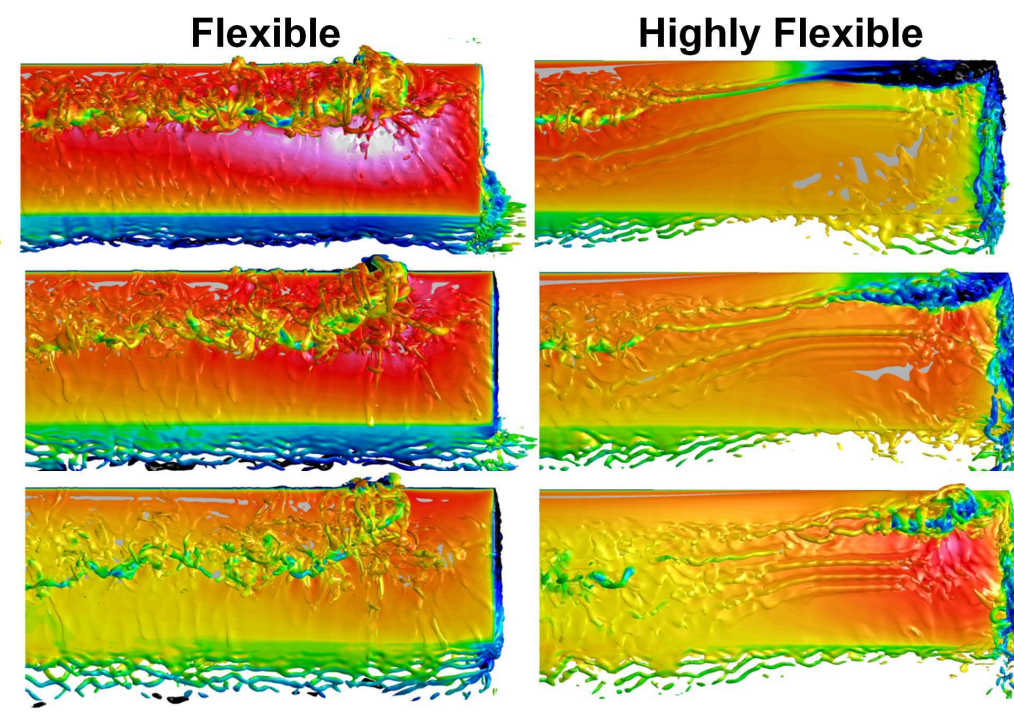

Figure 19. continued 


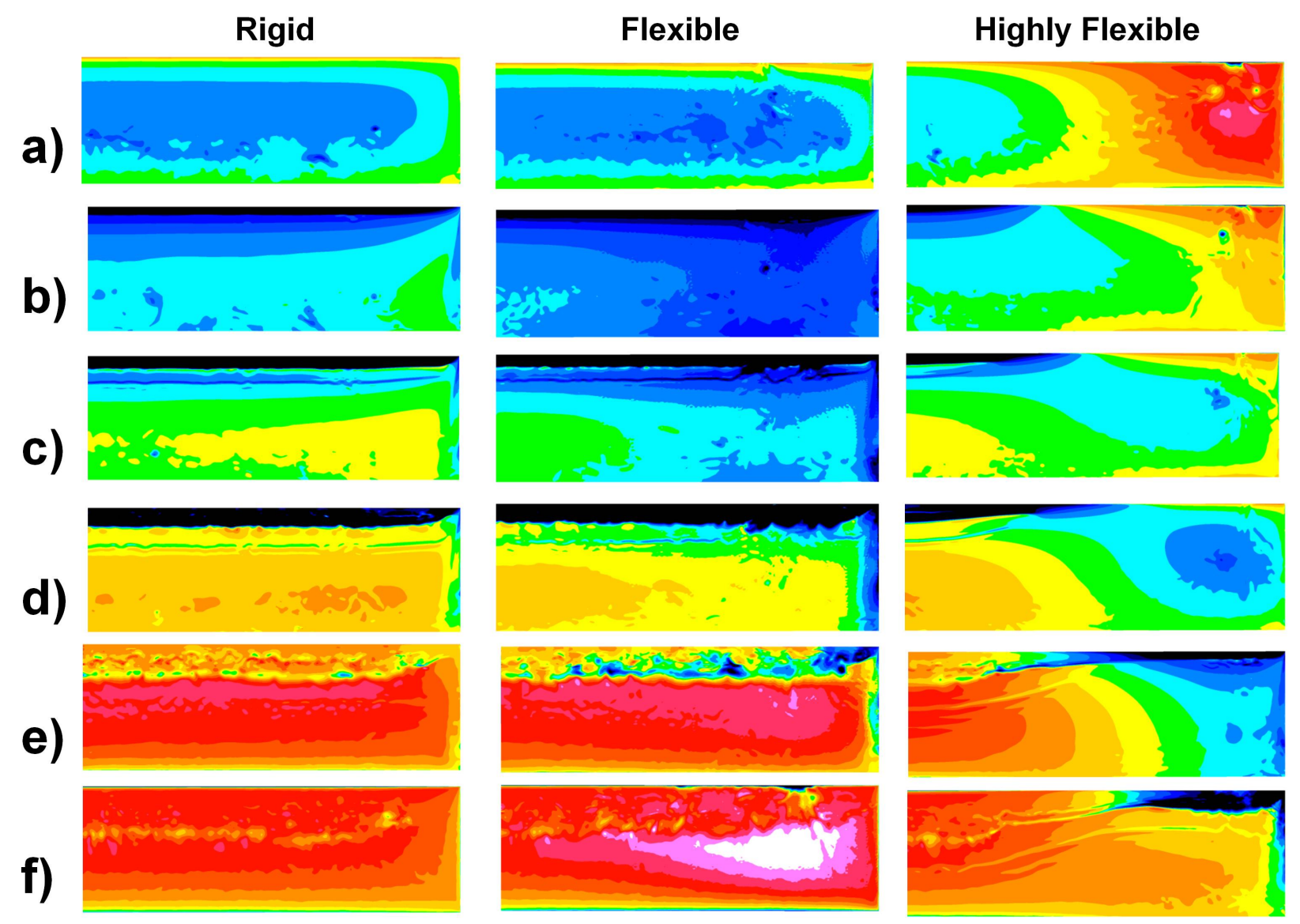

Figure 20. Comparison of Surface Pressure Coefficient on the Wing Upper Surface for the Rigid, Flexible and Highly-Flexible Cases

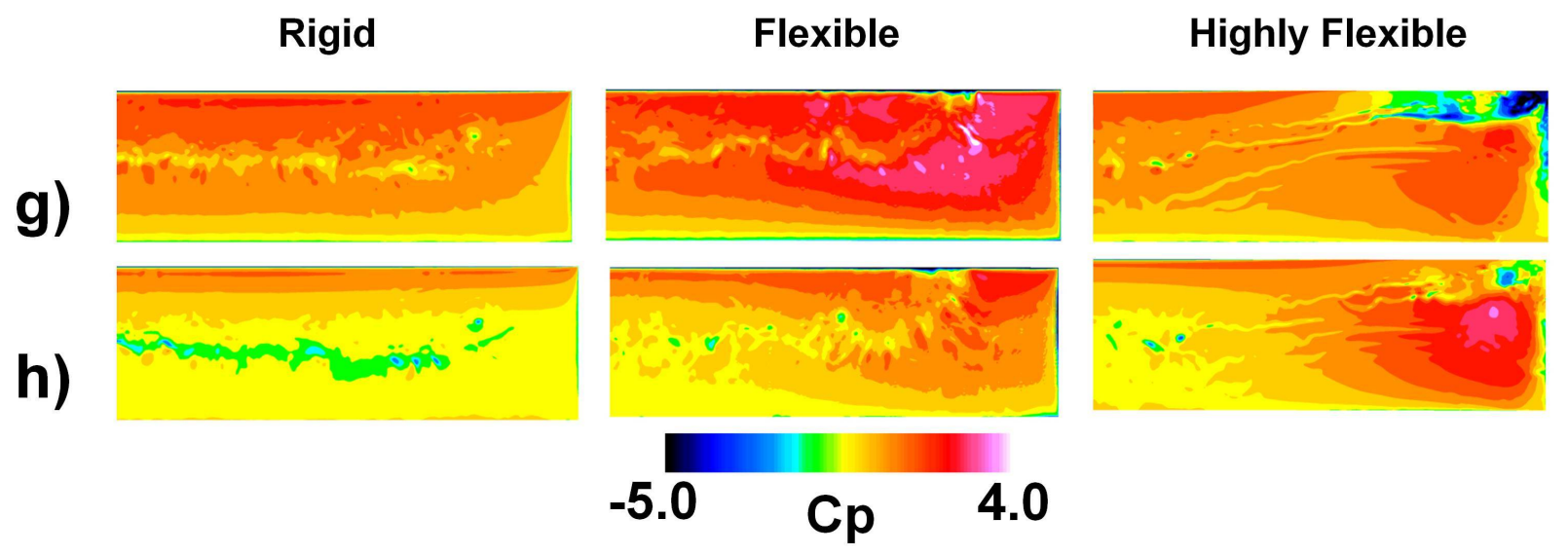

Figure 20. continued 
results in lower values of pressure underneath this vortex than what is obtained on the rigid wing. The wing tip vortices are also stronger for the flexible wing, Figures 19c-e. A corresponding low pressure band develops underneath the tip vortex, Figures 20c-e.

During the downstroke a laminar leading edge vortex system emerges on the upper surface, Figures 19b,c. As the wing moves from the midstroke to the bottom of the plunge motion, Figures 19c-e, this laminar vortex system undergoes flow transition with a fully transitional flow present at the bottom of the downstroke. A detailed description of this type of transition process for a typical wing section undergoing plunging motion may be found in Visbal. ${ }^{9}$ This transition process is enhanced for the flexible wing where the leading edge vortex is strengthened due to the larger effective angle of attack on the outboard portion of the wing, Fig. 18.

As the wing slows and reverses direction, the leading-edge vortex is shed convects downstream, Figs. 19e-h and 20e-h. The vortex on the rigid wing tends to remain fairly intact and two-dimensional until it approaches the trailing edge, Figures 19h,a. In contrast, the leading edge vortex on the flexible wing tends to break apart and be much more three-dimensional in character. Outboard on the flexible wing at $y \approx 2.22$ a large region of turbulent flow exists at the terminus of the leading edge vortex, Figure 19f. This region of turbulent flow persists in the leading edge region during the full upstroke of the wing and is only convected downstream after the wing commences the downstroke. This interesting behavior develops due to the large motion-induced negative pitch down that occurs for the flexible wing in this region. Figure 21 compares the

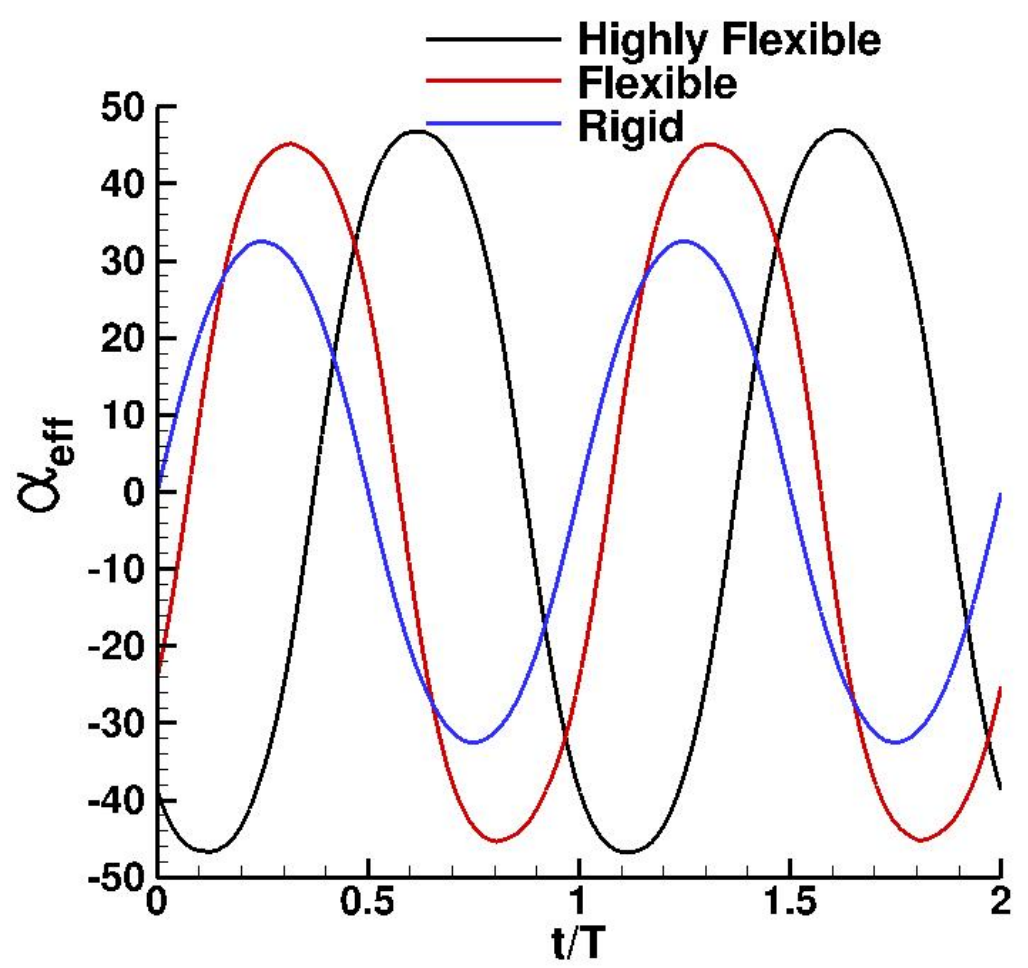

Figure 21. Temporal variation in the effective angle of attack at the wing tip for the rigid and flexible wings

time histories of the effective angle of attack at the wing tip for the rigid and flexible wings. During the portion of the wing upstroke from points $(\mathrm{e}, t / T=0.5)$ to $(\mathrm{h}, t / T=0.83)$ the flexible wing undergoes a more severe pitch down motion than the rigid wing from $\alpha_{\text {eff }}=24.5^{\circ}$ to $\alpha_{\text {eff }}=-45^{\circ}$. This rapid pitch down restricts the vorticity from propagating downstream. This feature is reminiscent of the flow behavior observed by $\mathrm{Visbal}^{9}$ for high frequency low amplitude plunge oscillations of a wing section.

A distinct high pressure zone is also noted on the flexible wing during the upstroke, Fig. 20f. This phenomena arises predominantly from the large deceleration and reversal of direction of the wing. Figure 22 compares the acceleration at the wing tip for the rigid and flexible cases. The peak acceleration, $d^{2} z / d t^{2}=$ 3.95 , occurs at $t / T=0.59$ and is 1.7 times the peak acceleration observed for the rigid case, $d^{2} z / d t^{2}=2.32$. These markedly higher accelerations as a result of the wing flexibility create greater noncirculatory effects 


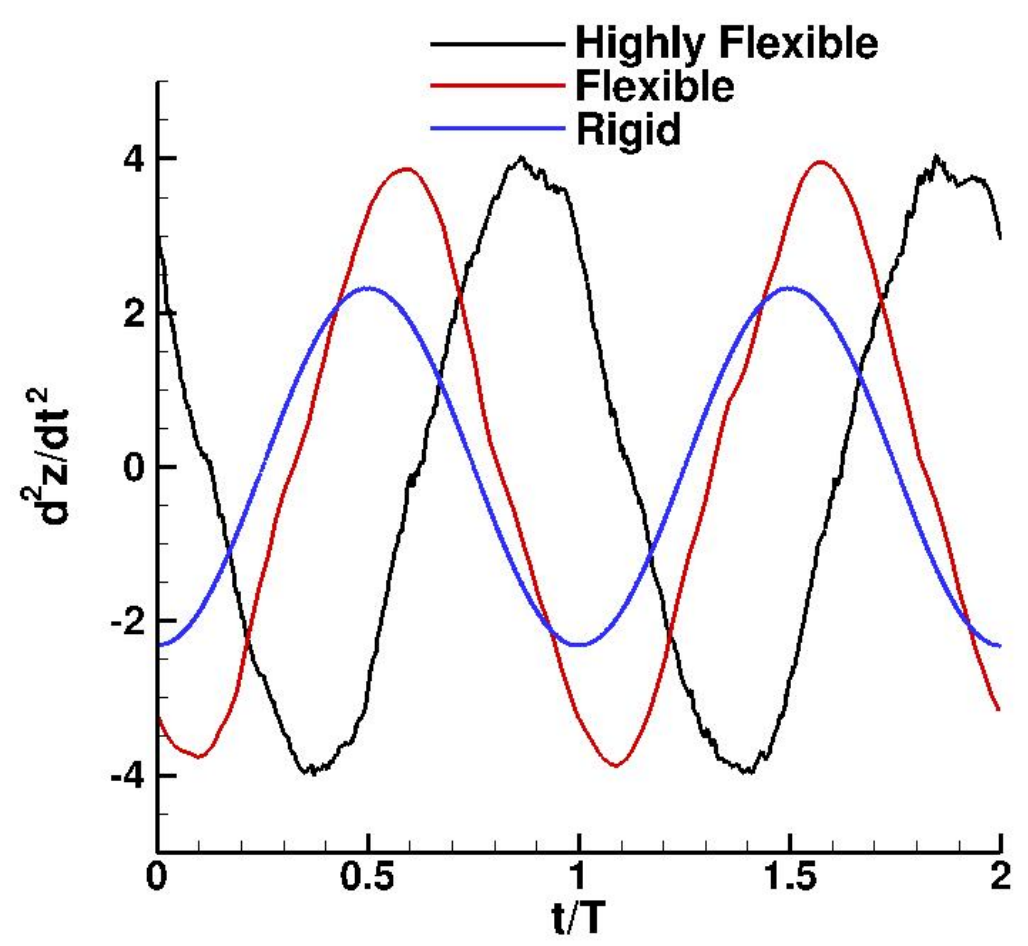

Figure 22. Temporal variation of acceleration at the wing tip for the rigid and flexible wings

(see Figure 8). This increase in the noncirculatory behavior results in the development of these higher pressure regions. Equivalent lower pressures outboard on the undersurface, Figure 20b, are also observed.

\section{Highly Flexible Case}

The maximum deflection (Table 2), effective angle of attack (Figure 21) and acceleration (Figure 22) at the wing tip do not vary significantly between the flexible and highly flexible case. It was noted in Figure 13 that there is a significant lag between the wing tip motion and the wing root motion for the highly flexible case, however. The wing tip deflection is out of phase with the wing root deflection for the majority of the plunge cycle. This leads to very large spanwise variations in effective angle of attack during the cycle. Figure 23 shows the spanwise variation in angle of attack for the three cases considered at the midpoint of the downward plunge, point $\mathrm{c}$ in Figure 3. The effective angle of attack for the highly flexible case exhibits a dramatic variation across the span from $32.5^{\circ}$ to $-38^{\circ}$. This notably alters the flowfield, Figs. 19 and 20.

Starting at the top and proceeding through the stroke cycle, Figure 19a-h, the development of the flowfield near the wing root is similar to the rigid and flexible cases. Further outboard on the wing the flowfield development differs greatly due to the large lag in the tip motion. At the top of the downstroke, Figure 19a, the wingtip is located below the wing root and is moving in an upward direction. The flowfield in the region of the wingtip at this point in the cycle is similar to the flexible case flowfield between points $\mathrm{f}$ and $\mathrm{g}$. Proceeding through the plunge motion with this phase offset the flow near the wingtip develops similarly to the flow for the flexible wing albeit with somewhat weaker flow features. This phase offset in flow development between the wing root and wing tip leads to portions of the cycle where the leading edge vortex is developing on the upper surface near the wing root and on the lower surface near the wing tip (see for instance Figure 19d). Substantial crossflow gradients in pressure are also present due to the large phase lag introduced by the greater flexibility.

Another interesting feature observed in the highly flexible case is the development of a series of small scale vortical structures in the boundary layer downstream of the leading edge vortex system during the time period when the wing locally undergoes a downward motion, Figures 19d-h. These vortices form initially 


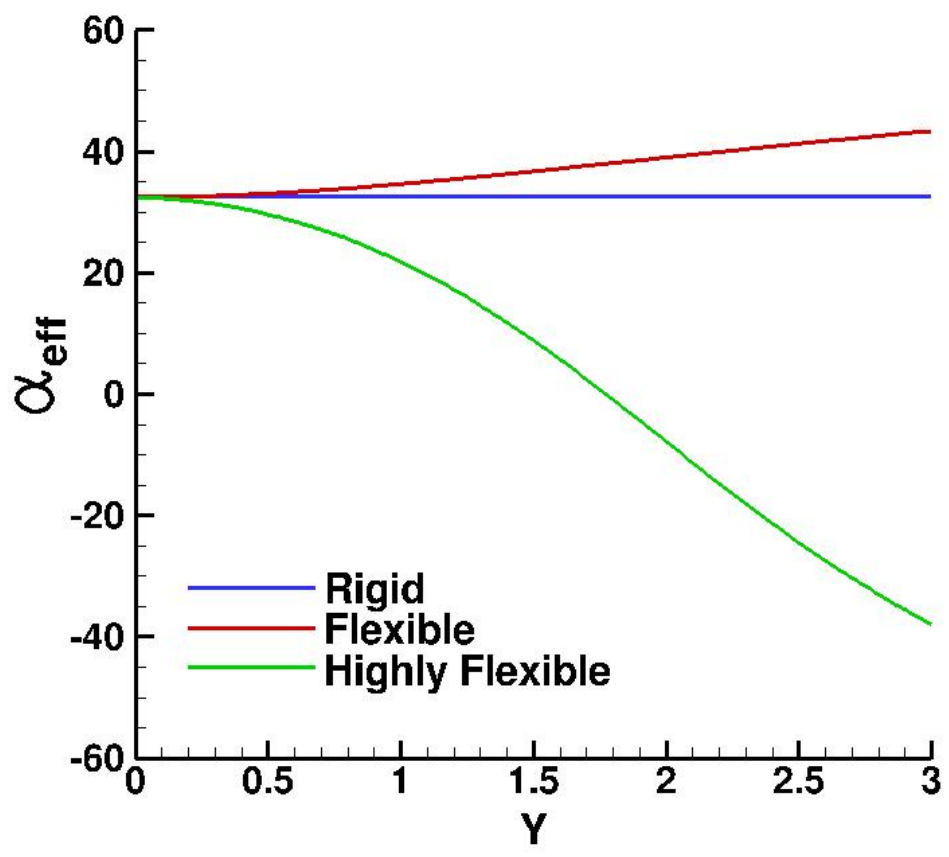

Figure 23. Spanwise variation of effective angle of attack at the midpoint of the downward plunge for the rigid, flexible and highly flexible cases

near the wing root and the development moves outboard on the wing as the plunge cycle progresses. In the case of the rigid and flexible wing an initial small scale vortex develops across the span, Figure 19d but this vortex rapidly breaks down with the onset of transition. In the highly flexible case a relief effect due to the large spanwise gradients tends to delay the onset of transition allowing this series of vortices to develop over a portion of the cycle. Eventually these vortices also succumb to transition.

The flowfield over the midportion of the wing develops quite differently from the rigid and flexible case. The development of the leading edge vortex in this region is much weaker, smaller in size and further downstream. This results from the fact that the maximum effective angle of attack at the midpsan, $\alpha_{\text {eff }} \approx$ $20^{\circ}$, for the highly flexible case is less than that for the rigid, $\alpha_{e f f}=32.5^{\circ}$, and flexible $\alpha_{\text {eff }} \approx 38^{\circ}$ response.

\section{Effect of Flexibility - Forces and Moments}

The experimental measurements of Heathcote ${ }^{4}$ demonstrated that the flexible case produces an enhanced thrust coefficient, while the highly flexible wing produces a very small amount of thrust. This effect is reproduced in these computations as seen in Figure 24. The mean thrust generated increases from $\overline{C T}=0.195$ for the rigid wing to $\overline{C T}=0.278$ for the flexible wing, an increase of $42 \%$. This is consistent with the experimentally reported increase in thrust of $50 \%$. The peak thrust generation and the corresponding maximum difference between the thrust for the rigid and flexible wing occurs over the midportion of the downstroke and upstroke. This corresponds to the portion of the motion where the formation of the leading edge vortex occurs. As discussed previously, the moderate flexibility of the wing leads to the formation of a stronger leading edge vortex which in turn leads to higher suction levels around the leading edge of the airfoil and therefore higher thrust.

A striking drop in the thrust produced is noted for the highly flexible wing. As was noted in the previous section, the large lag in the wing tip deflection considerably alters the flowfield development. An overall weaker leading edge vortex development occurs, in particular over the middle portion of the wing where the maximum effective angle of attack is greatly reduced. This disruption of the flowfield and alteration of the vortical flow development leads to a loss in thrust production. These results suggest that an optimal amount 


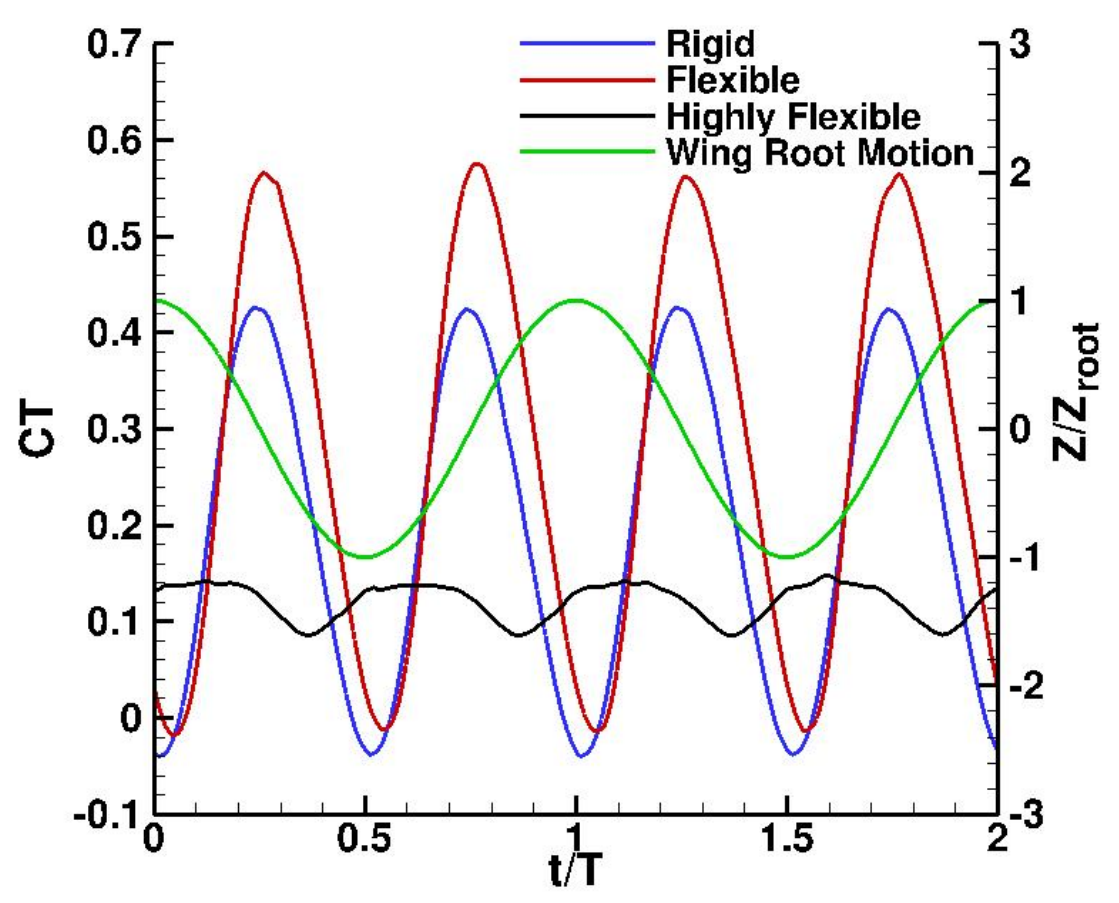

Figure 24. Effect of Flexibility on Generated Thrust Coefficient

of flexibility exists that would produce the most enhancement to thrust. This observation is concistent with the provious work of Heathcote et $\mathrm{al}^{4}$ and Chimakurthi. ${ }^{8}$

Enhanced lift is also obtained for the flexible wing. Figure 25 displays the time histories of the lift coefficient for the rigid, flexible and highly flexible wings. The peaks in the lift time histories correlate with the locations of maximum peak accelerations, Figure 22. The higher peak lift in the flexible case is consistent with the significantly larger peak accelerations. These greater accelerations give rise to stronger noncirculatory loads that arise from the previously described high and low pressure zones. The additional flexibility introduced in the highly flexible case results in maximum lift coefficients similar to the rigid case. This result again suggest that there is an optimum flexibility for lift production.

The impact of flexibility on the moment coefficients about the quarter chord point is also investigated. The rolling moment (considering only the half-wing computed) and pitching moment are plotted in Figures 26 and 27 respectively. Both the pitch and roll moment coefficient vary with the same frequency but lag the plunge motion. The flexible wing has higher peak values of both the rolling and pitching moment that occur during the initial portion of the upstroke and downstroke. The enhancement of these moments is associated with the development of the high pressure zone seen in Fig. 20f and a corresponding lower pressure on the opposite surface, Fig. 20b. The yawing moment (again only for the half-wing computed), Figure 28, exhibits a frequency similar to the thrust coefficient. As in the case of the thrust coefficient, the higher values of yawing moment in the flexible case are attributable to the strengthening of the leading edge vortex due to the increased effective angle of attack that results from the flexibility of the wing. The increased flexibility of the wing in the highly flexible case again results in a dropoff in the levels of all three moments due to the modification of the flowfield that results from the large phase lag introduced.

\section{Conclusions}

High fidelity computations for a rigid and plunging flexible wings have been performed. The computational solver used couples a high-order Navier-Stokes solver with a geometrically nonlinear structural solver 


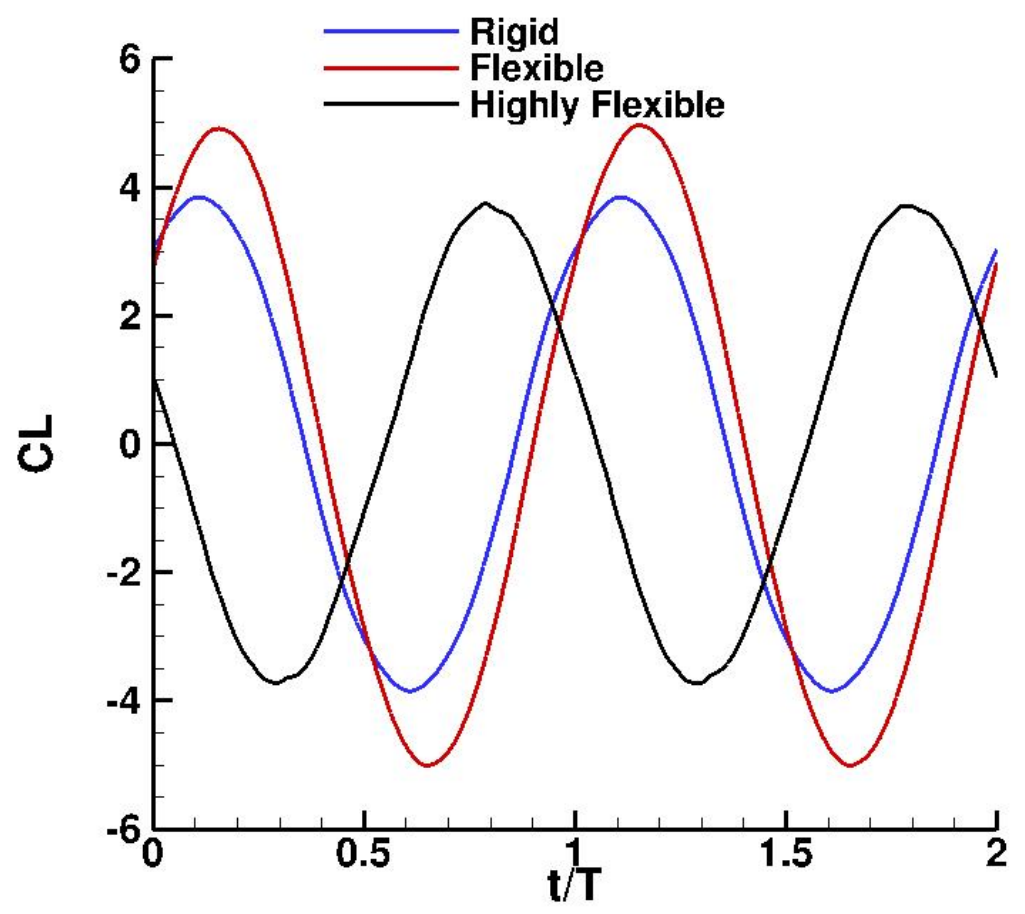

Figure 25. Effect of Flexibility on Generated Lift Coefficient

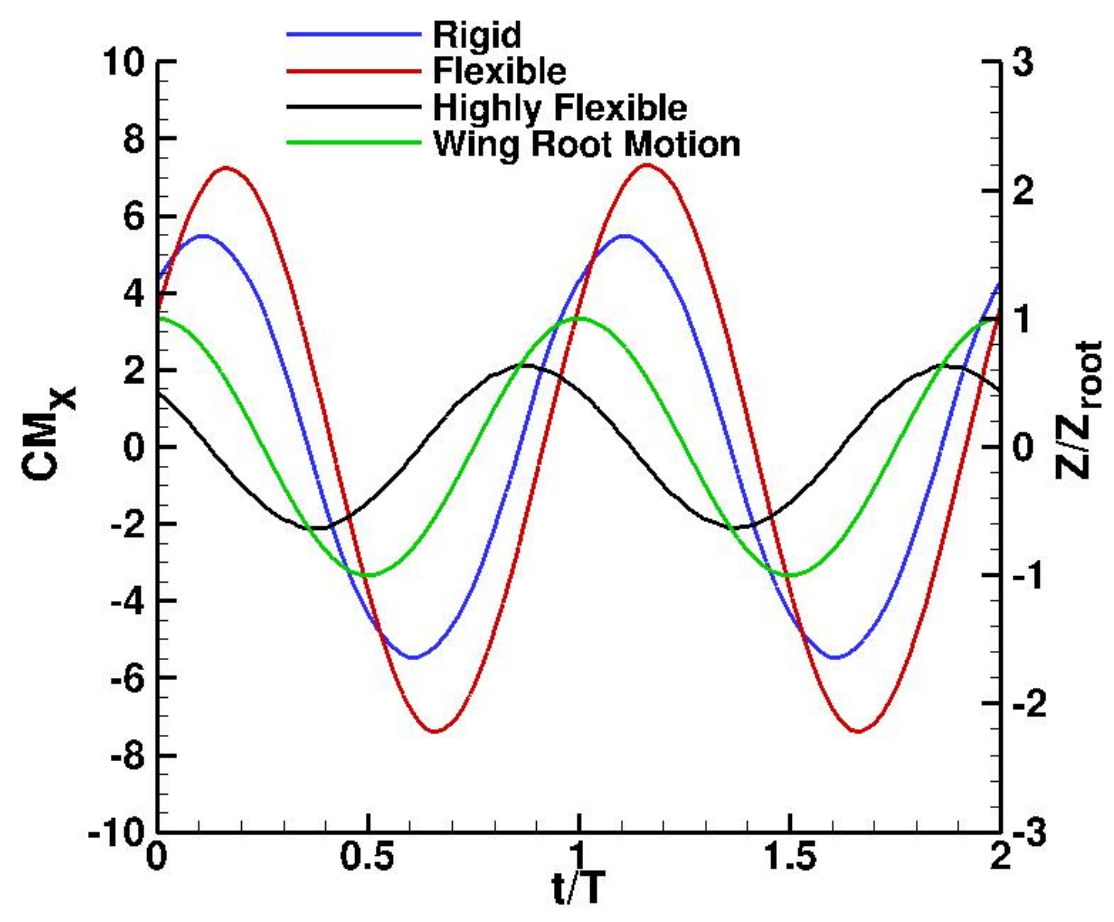

Figure 26. Effect of Flexibility on Rolling Moment Coefficient 


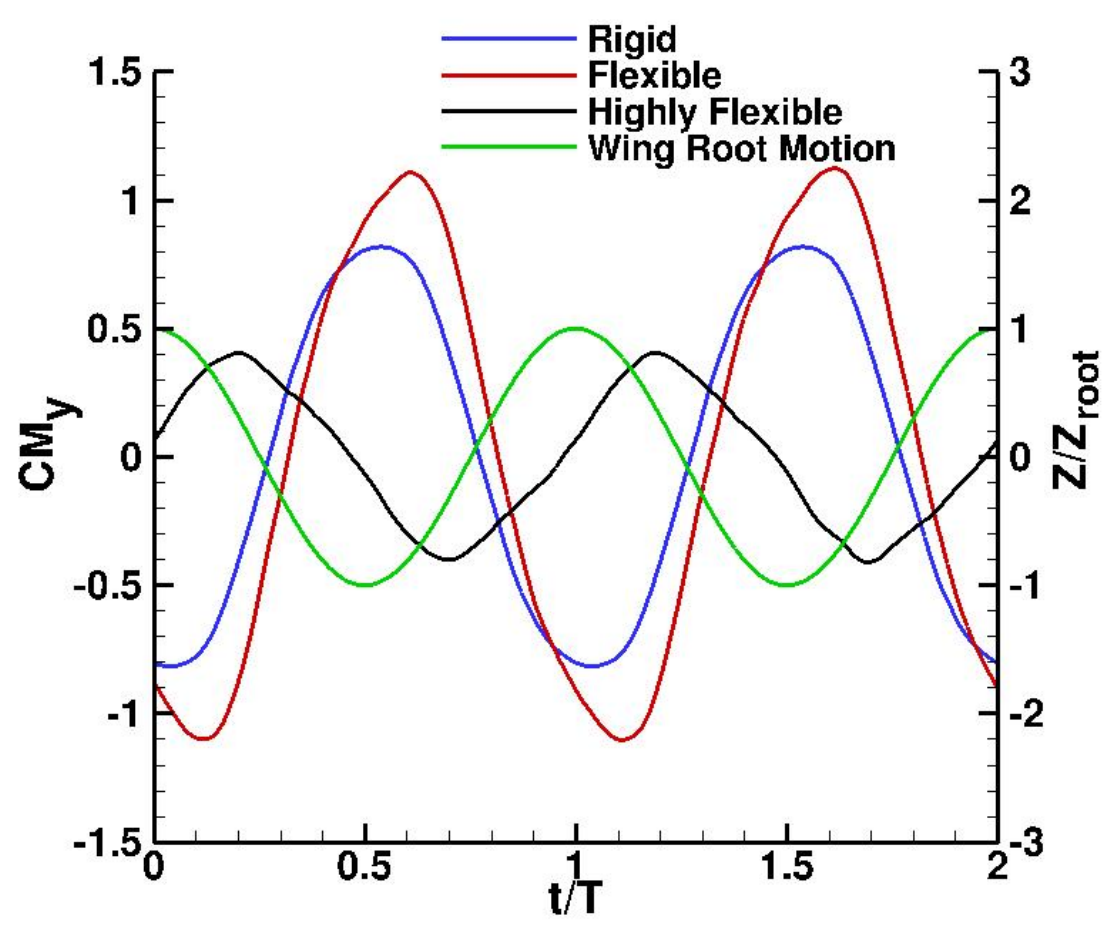

Figure 27. Effect of Flexibility on Pitching Moment Coefficient

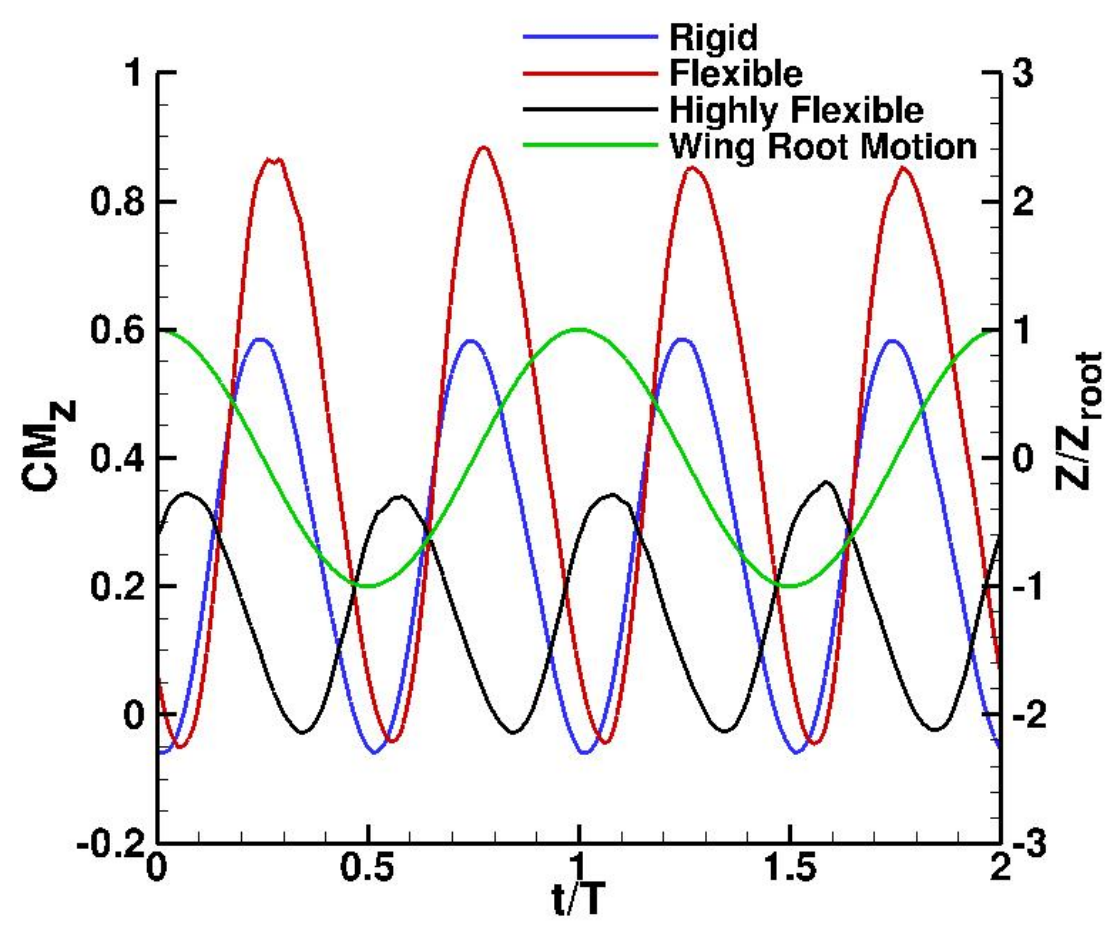

Figure 28. Effect of Flexibility on Yawing Moment Coefficient 
based on the dynamics of a general three-dimensional anisotropic slender solid. Computed results are compared with available experimental measurements demonstrating good agreement between the computation and experiment.

The longitudinal flexibility of the wing gives rise to significant spanwise variations in the wing deflection, motion-induced effective angle of attack and wing acceleration. These effects produce a complex interaction between the vortex dynamics and the structural motion. For the moderately flexible wing higher effective angles of attack achieved result in the development of a stronger leading edge vortex system outboard on the wing. Towards the tip of the wing the more rapid effective pitch down and pitch up motions inhibit the convection of the leading edge vorticity. The larger accelerations outboard produce corresponding regions of higher and lower pressure on the wing upper and lower surfaces due inviscid effects.

As flexibility is increased in the highly flexible case, a significant lag between the wing tip and wing root motions develops that changes the character of the vortex dynamics over the wing. Inboard towards the wing root the flow develops in a similar manner to the rigid and moderately flexible cases. Out board towards the wing tip the flow development is reminiscent of the moderately flexible wing with an appropriate phase shift. Over the mid portion of the wing the strength of the leading edge vortex system is reduced due to the much smaller effective angles of attack achieved. During certain portions of the motion, the leading edge vortex is split between the upper and lower surfaces.

The spanwise variation and increased strength of the leading edge vortex system in the moderately flexible case produces enhanced thrust as well as larger yawing moments. Increased noncirculatory loads due to the larger values of acceleration achieved by the moderately flexible wing result in higher peak lift, rolling moment and pitching moment coefficients. The positive effects for moderate flexibility are lost as flexibility is increased in the highly flexible case. These results suggest that an optimum amount of flexibility exists for the case of a plunging wing and is associated with wing motions where the wing tip and wing root motions are in phase over much of the plunge cycle.

\section{Acknowledgments}

This work was sponsored by AFOSR under a task monitored by Dr. Doug Smith and supported in part by a grant of HPC time from the DoD HPC Shared Resource Centers at the Air Force Research Laboratory, Wright-Patterson AFB, OH and the Army Corps of Engineers Research and Development Center at Vicksburg, MS. The authors wish to thank Drs. Gursul, Wang and Heathcote of the University of Bath for providing the experimental data. The first author acknowledges several helpful discussions with dr. Miguel Visbal. The authors at the University of Michigan were supported in part by an AFOSR Multidisciplinary University Research Initiative (MURI) grant and by the Michigan/AFRL/Boeing Collaborative Center in Aeronautical Sciences.

\section{References}

\footnotetext{
${ }^{1}$ Shyy, W., Lian, Y., Tang, J., Viieru, D., and Liu, H., Aerodynamics of Low Reynolds Number Flyers, Cambridge University Press, New York, 2008.

${ }^{2}$ Mueller, T. J., Fixed and Flapping Wing Aerodynamics for Micro Air Vehicle Applications, Vol. 195 of Progress in Aeronautics and Astronautics, AIAA, New York, 2001.

${ }^{3}$ Shyy, W., Ifju, P., and Viieru, D., "Membrane Wing-Based Micro Air Vehicles," Applied Mechanics Review, Vol. 58, No. 1-6, 2005, pp. 283-301.

${ }^{4}$ Heathcote, S., Wang, Z., and Gursul, I., "Effect of Spanwise Flexibility on Flapping Wing Propulsion," Journal of Fluids and Structures, Vol. 24, No. 2, 2008, pp. 183-199.

${ }^{5}$ Tang, J., Chimakurthi, S., Palacios, R., Cesnik, C. E. S., and Shyy, W., "Computational Fluid-Structure Interaction of a Deformable Flapping Wing for Micro Air Vehicle Applications," AIAA-2008-0615, January 2008.

${ }^{6}$ Chimakurthi, S., Tang, J., Palacios, R., Cesnik, C. E. S., and Shyy, W., "Computational Aeroelasticity Framework for Analyzing Flapping Wing Micro Air Vehicles," AIAA Journal, Vol. 47, No. 8, 2009, pp. 1865-1878.

${ }^{7}$ Aono, H., Chimakurthi, S., , Cesnik, C. E. S., Liu, H., and Shyy, W., "Computational Modeling of Spanwise Flexibility Effects on Flapping Wing Aerodynamics," AIAA-2009-1270, January 2009.

${ }^{8}$ Chimakurthi, S. K., "A Computational Aeroelastic Framework for Analyzing Flapping Wings," Doctoral Dissertation of the University of Michigan, 2009.

${ }^{9}$ Visbal, M. R., "High-Fidelity Simulation of Transitional Flows Past a Plunging Airfoil," AIAA Journal, Vol. 47, No. 11, November 2009, pp. 2685-2697.

${ }^{10}$ Gordnier, R. E., "Implicit LES Simulations of a Low Reynolds Number Flexible Memrane Wing Airfoil," AIAA-2009-0579, January 2009.
} 
${ }^{11}$ Anderson, D., Tannehill, J., and Pletcher, R., Computational Fluid Mechanics and Heat Transfer, McGraw-Hill Book Company, 1984.

${ }^{12}$ Lele, S., "Compact Finite Difference Schemes with Spectral-like Resolution," Journal of Computational Physics, Vol. 103, 1992, pp. 16-42.

${ }^{13}$ Gaitonde, D. and Visbal, M., "High-Order Schemes for Navier-Stokes Equations: Algorithm and Implementation into FDL3DI," Tech. Rep. AFRL-VA-WP-TR-1998-3060, Air Force Research Laboratory, Wright-Patterson AFB, 1998.

${ }^{14}$ Visbal, M. and Gaitonde, D., "High-Order Accurate Methods for Unsteady Vortical Flows on Curvilinear Meshes," AIAA Paper 98-0131, January 1998.

${ }^{15}$ Gaitonde, D., Shang, J., and Young, J., "Practical Aspects of High-Order Accurate Finite-Volume Schemes for Electromagnetics," AIAA Paper 97-0363, Jan. 1997.

${ }^{16}$ Visbal, M. and Gaitonde, D., "High-Order Accurate Methods for Complex Unsteady Subsonic Flows," AIAA Journal, Vol. 37, No. 10, 1999, pp. 1231-1239.

${ }^{17}$ Alpert, P., "Implicit Filtering in Conjunction with Explicit Filtering," J. Comp. Phys., Vol. 44, 1981, pp. 212-219.

${ }^{18}$ Gaitonde, D. and Visbal, M., "Further Development of a Navier-Stokes Solution Procedure Based on Higher-Order Formulas," AIAA Paper 99-0557, January 1999.

${ }^{19}$ Beam, R. and Warming, R., "An Implicit Factored Scheme for the Compressible Navier-Stokes Equations," AIAA Journal, Vol. 16, No. 4, 1978, pp. 393-402.

${ }^{20}$ Jameson, A., Schmidt, W., and Turkel, E., "Numerical Solutions of the Euler Equations by a Finite Volume Method Using Runge-Kutta Time Stepping Schemes," AIAA Paper 81-1259, 1981.

${ }^{21}$ Pulliam, T., "Artificial Dissipation models for the Euler Equations," AIAA, Vol. 24, No. 12, 1986, pp. 1931-1940.

${ }^{22}$ Pulliam, T. and Chaussee, D., "A Diagonal Form of an Implicit Approximate-Factorization Algorithm," Journal of Computational Physics, Vol. 39, No. 2, 1981, pp. 347-363.

${ }^{23}$ Visbal, M. R., Morgan, P. E., and Rizzetta, D. P., "An Implicit LES Approach Based on High-Order Compact Differencing and Filtering Schemes (Invited)," AIAA-2003-4098, June 2003.

${ }^{24}$ Visbal, M. and Gaitonde, D., "Very High-Order Spatially Implicit Schemes for Computational Acoustics on Curvilinear Meshes," Journal of Computational Acoustics, 2001.

${ }^{25}$ Palacios, R. and Cesnik, C. E. S., "A Geometrically Nonlinear Theory of Active Composite Beams with Deformable Cross Sections," AIAA Journal, Vol. 46, No. 2, 2008, pp. 439-450.

${ }^{26}$ Palacios, R. and Cesnik, C. E. S., "Cross-Sectonal Analysis of Non-homogenous Anistropic Active Slender Structures," AIAA Journal, Vol. 43, No. 12, 2005, pp. 2624-2638.

${ }^{27}$ Cesnik, C. E. S. and Hodges, D. H., "VABS: A New Concept for Composite Rotor Blade Cross-Sectional Modeling," Journal of the American Helicopter Society, Vol. 42, No. 1, 1997, pp. 27-38.

${ }^{28}$ Smith, M. J., Hodges, D., and Cesnik, C. E. S., "Evaluation of Computational Algorithms Suitable for Fluid-Structure Interactions," Journal of Aircraft, Vol. 37, No. 2, 2000, pp. 282-294.

${ }^{29}$ Melville, R. B., Morton, S. A., and Rizzetta, D. P., "Implementation of a Fully-Implicit, Aeroelastic Navier-Stokes Solver," AIAA 97-2039, June 1997.

${ }^{30}$ Fung, Y., An Introduction to the Theory of Aeroelasticity, Dover, 1993. 